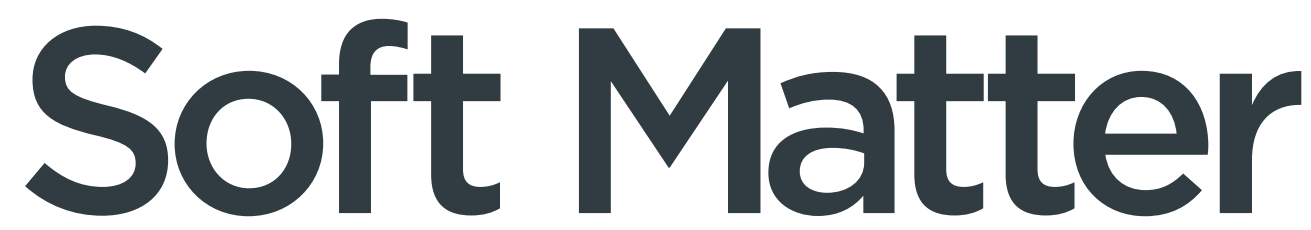

Volume 17

Number 9

7 March 2021

Pages 2343-2676

rsc.li/soft-matter-journal

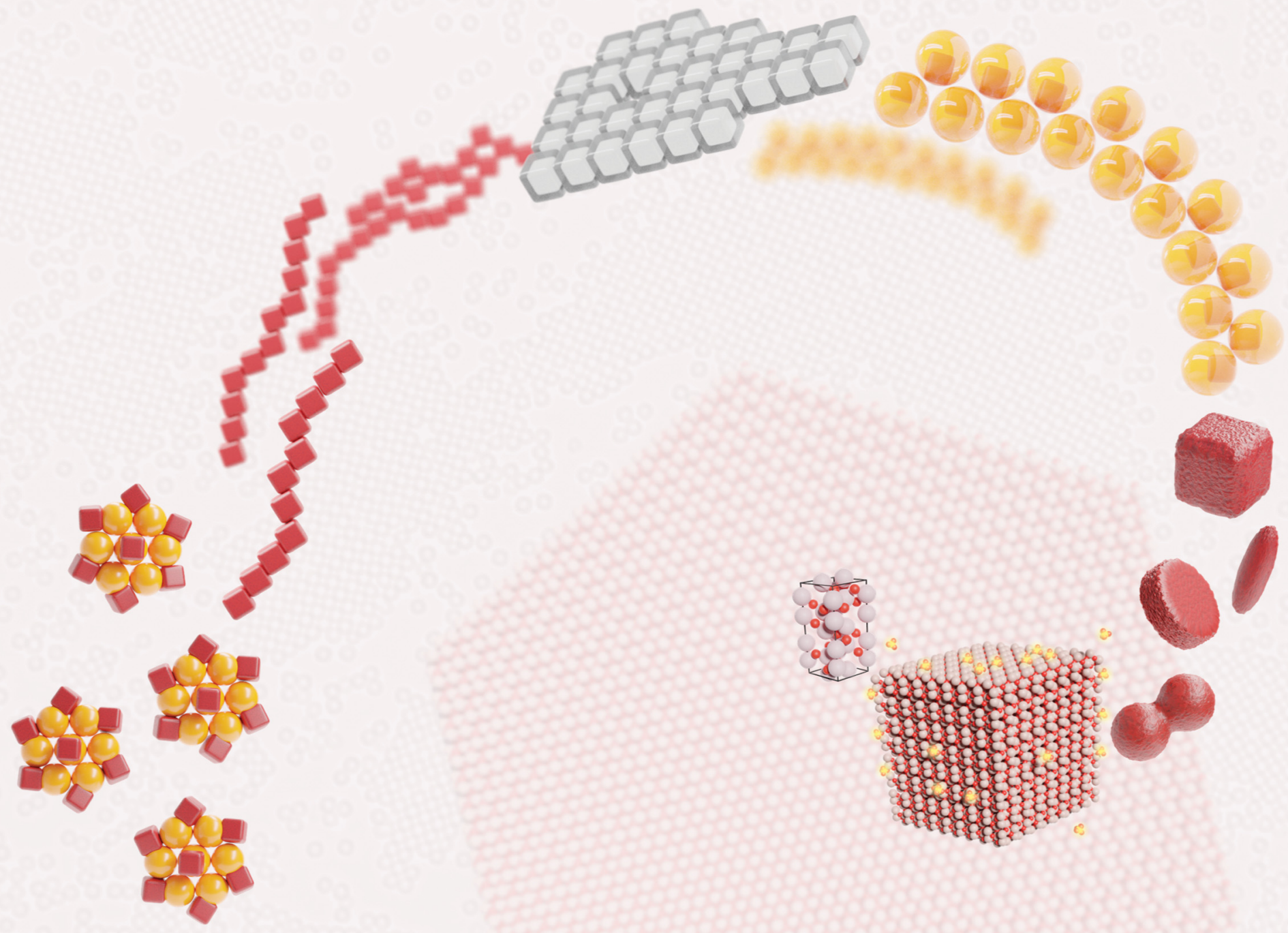

\section{REVIEW ARTICLE}

J. M. Meijer and L. Rossi

Preparation, properties, and applications of magnetic hematite microparticles 
Check for updates

Cite this: Soft Matter, 2021,

17,2354

Received 6th November 2020,

Accepted 21st January 2021

DOI: 10.1039/d0sm01977a

rsc.li/soft-matter-journal

\title{
Preparation, properties, and applications of magnetic hematite microparticles
}

\author{
J. M. Meijer (iD ${ }^{a}$ and L. Rossi (D)*b
}

\begin{abstract}
Hematite microparticles are becoming increasingly important components in the soft matter field. The remarkable combination of magnetic and photocatalytic properties that characterize them, coupled with the variety of uniform and monodisperse shapes that they can be synthesized in, makes them a one of a kind colloidal model system. Thanks to these properties, hematite microparticles have been recently applied in several important soft matter applications, spanning from novel colloidal building blocks for self-assembly to necessary tools to investigate and understand fundamental problems. In this review article we provide a detailed overview of the traditional methods available for the preparation of hematite microparticles of different shapes, devoting special attention on some of the most common hiccups that could hider a successful synthesis. We furthermore review the particles' most important physico-chemical properties and their most relevant applications in the soft matter field.
\end{abstract}

\section{Introduction}

Hematite microparticles with uniform shape and size have been available since the first method for their controlled synthesis was introduced by Matijevic and Scheiner in the late $70 \mathrm{~s}^{1}$ in an article that directly reported the preparation

${ }^{a}$ Department of Applied Physics, Eindhoven University of Technology, 5600 MB Eindhoven, The Netherlands

${ }^{b}$ Department of Chemical Engineering, Delft University of Technology, 2629 HZ Delft, The Netherlands. E-mail: L.Rossi@tudelft.nl methods of various shapes. Since then, the introduction of a high yield synthesis procedure via a gel-sol method ${ }^{2}$ has made hematite microparticles available for a variety of applications. However, it is only more recently that scientists have started to take full advantage of this colloidal system. One of the main reasons for the current interest in magnetic hematite particles lies in their unique magnetic properties which sets them apart from other commonly available magnetic materials. The magnetic nature of hematite microparticles was first identified in the $1980 \mathrm{~s},{ }^{3,4}$ when typical dipolar structure formation of hematite particles were first reported from optical microscopy

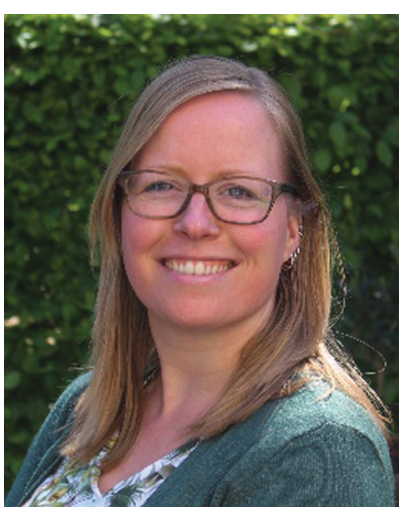

J. M. Meijer
Janne-Mieke Meijer obtained her $P h D$ in Physical and Colloid Chemistry from Utrecht University in 2015. She then joined Lund University for a postdoc, followed by a Humboldt fellowship at the University of Konstanz. Early 2019, she returned to the Netherlands with a Veni fellowship at the University of Amsterdam. Mid-2020, she joined the Applied Physics Department of Eindhoven University of Technology as Assistant Professor in Experimetal Soft Matter. Her current research focusses on complex colloidal systems to discover the fundamental principles of how building block design influences self-organization and how to control the self-assembly process to engineer new materials.

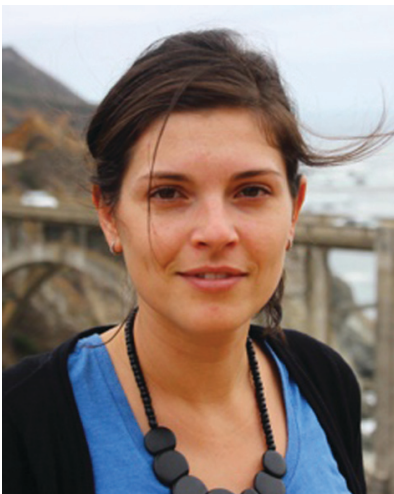

L. Rossi
Laura Rossi obtained her PhD in Physical and Colloid Chemistry from Utrecht University in 2012. After postdocs at the University of California, Los Angeles and at the University of Amsterdam, she joined the Chemical Engineering Department at TU Delft where she leads the group on Colloidal Engineering and Materials Design as Assistant Professor. Her research work is set at the interface of chemistry, physics and engineering and currently focuses on the design of complex magnetic colloidal particles for the self-assembly of novel materials. She is actively involved in outreach activities with local high schools to promote STEM subjects among students. 
observations. At that point however, not much was known about their specific magnetic properties. ${ }^{5,6}$

Now we know that hematite is a canted antiferromagnet ${ }^{7}$ with a spontaneous magnetization that is much lower than that of the more commonly used magnetite, but because its magnetic domain is a few micrometer in size, ${ }^{8}$ we can use it to prepare micron-sized particles with a strong permanent dipole moment. This unique aspect allows the use of hematite microparticles as model systems in magnetic assembly and manipulation $^{9-14}$ and as magnetic components for the design of more complex building blocks for self-assembly. ${ }^{15,16}$ Beside self-assembly, these microparticles can be used to devise novel systems that show interesting new physical phenomena generated by applying rotating magnetic fields, such as microrollers of composite hematite-polymer particles, ${ }^{17}$ and, more recently, colloidal chiral fluids, ${ }^{18}$ and reconfigurable magnetic swarms. ${ }^{19}$ Another reason for hematite microparticles' growing appeal, is their high yield syntheses that can lead to a variety of different anisotropic shapes, making them ideal templates for the preparation of amorphous silica particles, ${ }^{20-26}$ and thus allowing bulk synthesis of well-defined anisotropic particles for the study of shape-directed self-assembly. Among one of the most interesting properties of hematite for soft matter applications is its photocatalytic activity. This property has been recently successfully exploited for the design and production of well-defined colloidal swimmers with real scale-up production potential. ${ }^{27-31}$ Hematite is a cheap and abundant material and its unique physicochemical and magnetic properties in the colloidal form provide the soft matter community with an exceptional system that possess all the features for the design of novel classes of active systems that can be chemically or magnetically actuated.

In this article we are not aiming at giving an exhaustive review of all the hematite colloidal systems developed to date. This article is intentionally focused on the preparation, properties and applications of hematite particles with uniform shape and size in the micron-size range. This is not to take away from all the works that have been done over the years on hematite nanoparticles, especially in relation to particle synthesis, ${ }^{32-43}$ but because hematite microparticles appear to be better suited for a lot of soft matter applications in which magnetic interactions and single particle visualization become essential. We furthermore pay special attention to the preparation methods to provide a guide, also to the non-chemist, to facilitate particle synthesis and therefore their applications.

\section{Preparation of micron-sized hematite particles}

\subsection{Synthesis methods}

Due to its thermodynamic stability compared to other iron oxides, hematite is easily precipitated from iron(III) solutions. ${ }^{44}$ There are various synthesis methods available to prepare micron sized hematite particles uniform in shape and with a narrow size distribution. The most commonly used are forced hydrolysis $^{1}$ (also called hydrothermal synthesis) and gel-sol processing. ${ }^{2}$

In the forced hydrolysis method an iron(III) salt solution with a "dilute" concentration (typically $10^{-3}$ to $10^{-2} \mathrm{M}$ ) is (acidified and) aged at temperatures between $80-150{ }^{\circ} \mathrm{C}$. This process forces the hydrolysis of iron(III) as the deprotonation of coordinated water is greatly accelerated with increasing temperature. The hydrolysis product, $\mathrm{Fe}(\mathrm{OH})_{3}$, is the intermediate for precipitation of iron(hydrous)oxide and when generated at a proper rate, eventually uniform hematite $\left(\alpha-\mathrm{Fe}_{2} \mathrm{O}_{3}\right)$ particles are obtained. The shape, size and nature of the particles depends on the concentration, $\mathrm{pH}$, temperature, time of ageing and the presence of other anions or molecules in solution. Establishing this method and identifying the specific regions that yield uniform hematite particles was described by Matijević and Scheiner as "a rather arduous task". ${ }^{1}$ For instance, when employing both $\mathrm{Fe}\left(\mathrm{NO}_{3}\right)_{3}$ and $\mathrm{Fe}\left(\mathrm{ClO}_{4}\right)_{3}$ as starting salts, the resulting particles are pure hematite, while when starting with $\mathrm{FeCl}_{3}$ either uniform akaganéite $(\beta-\mathrm{FeOOH})$ particles or hematite particle are obtained depending on the exact concentration of $\mathrm{Fe}$ and $\mathrm{Cl}$ ions. Subsequent studies have addressed the role of other additives, such as different alcohols, ${ }^{45,46}$ glycine ${ }^{47}$ and amines, ${ }^{48}$ as well as the presence of polymers. ${ }^{49-51}$ Interestingly, as the hematite precipitation is so robust, micron-sized hematite particles have also shown up in several studies involving the heating of dilute aqueous iron solutions to high temperatures $\left(150-180{ }^{\circ} \mathrm{C}\right)$. In these studies the method is often referred to as hydrothermal synthesis or processing $^{52}$ and is often reported coupled with additives, such as ionic liquids, ${ }^{53}$ ammonia, ${ }^{54}$ caffeine, ${ }^{55}$ ethylene glycol, ${ }^{56}$ and urea and saccharide. ${ }^{57}$ These syntheses simply represent a variation of the forced hydrolysis process and many of the proposed explanations for particle formation in these studies are not correct, for example, suggesting oriented attachment of nano-particles or claiming that the additive drives the micronsized particle formation. We report a more detailed discussion on the exact formation mechanism in the forced hydrolysis method and the role of additives in Sections 2.2 and 2.3.

The gel-sol method was first developed by Sugimoto, ${ }^{2}$ inspired by the observation that hydroxide gels had led to the formation of uniform cobalt(II,III)oxide and magnetite particles. For the formation of hematite, the method involves mixing a concentrated $(2.0 \mathrm{M})$ aqueous solution of $\mathrm{FeCl}_{3}$ with an aqueous solution of sodium hydroxyde $(\mathrm{NaOH})$ of even higher concentration $(6.0 \mathrm{M})$ to obtain a condensed iron hydroxide gel that is subsequently aged undisturbed for 8 days at $100{ }^{\circ} \mathrm{C}$. The network of the hydroxide gel acts as a "scaffold" to the hematite particles during their growth preventing unwanted random coagulation. The gel also serves as a reservoir for hydrolyzed iron species. Through a two-step phase transformation via rod-like $\beta$-FeOOH particles, the $\mathrm{Fe}(\mathrm{OH})_{3}$ is transformed into uniform $\alpha-\mathrm{Fe}_{2} \mathrm{O}_{3}$ particles in large quantities with yields close to $100 \%$. To be more specific, starting with $200 \mathrm{~mL}$ reaction mixture a total of $>7 \mathrm{~g}$ of particles can be obtained. This is the reason the gel-sol method has been employed in most of the studies described in later sections focusing on the 
unique behavior of dense or large particle systems. Follow-up studies have explored the role of adding different anions to the synthesis, including sulphates and phosphates, ${ }^{58}$ and hydroxide ions ${ }^{59}$ as well as organic additives. ${ }^{60}$

The synthesis methods discussed so far, are strategies to obtain micron sized hematite particles. However, it is important to note that an abundance of work has been done on the preparation of hematite nanoparticles (i.e. particles with dimensions smaller than $\sim 200 \mathrm{~nm}$ ). Including the synthesis of hematite spindles or nanocubes that can be obtained with the forced hydrolysis synthesis at low concentrations of $\mathrm{FeCl}_{3}(0.01-0.02 \mathrm{M}) \cdot{ }^{33,41-43}$ In addition, nanoparticles can be prepared in different ways, such as via hydrothermal decomposition. ${ }^{35,36,38,61}$

\subsection{Shape control}

The unique nature of the hematite particle synthesis is that a variety of different shapes can be obtained. Fig. 1 shows an overview of several of these different hematite shapes from a collection of works. These shapes include spheres, ${ }^{1}$ double spheres, ${ }^{47}$ ellipsoids, ${ }^{33,58}$ double ellipsoids, ${ }^{1,32}$ cubes, ${ }^{1,2}$ peanuts, ${ }^{58}$ disks or platelets, ${ }^{46,49,62,63}$ hexagonal platelets ${ }^{59}$ and bipyramids. ${ }^{1}$ Table 1 provides an overview of the different shapes and some of the synthesis conditions through which these shapes can be obtained. We again note that we have included only those procedures that allow the preparation of monodisperse particles in the micron size range with uniform shape.

The decisive factor to control the final particle shape is the nature of the specific anions or additives that are added to the iron(III) solutions ${ }^{65}$ during synthesis, which is most easily demonstrated by looking at the different shapes obtained with the gel-sol method. To create the iron hydroxide gel, the gel-sol method always involves concentrated $\mathrm{FeCl}_{3}$ and $\mathrm{NaOH}$ solutions. Without additives, cubic particle are obtained. ${ }^{2}$ When adding inorganic anions other shapes are obtained: ellipsoidal and peanut shapes with sulfate or phosphate, ${ }^{58}$ and plate-like particles with hydroxide ions. ${ }^{59}$ The mechanism
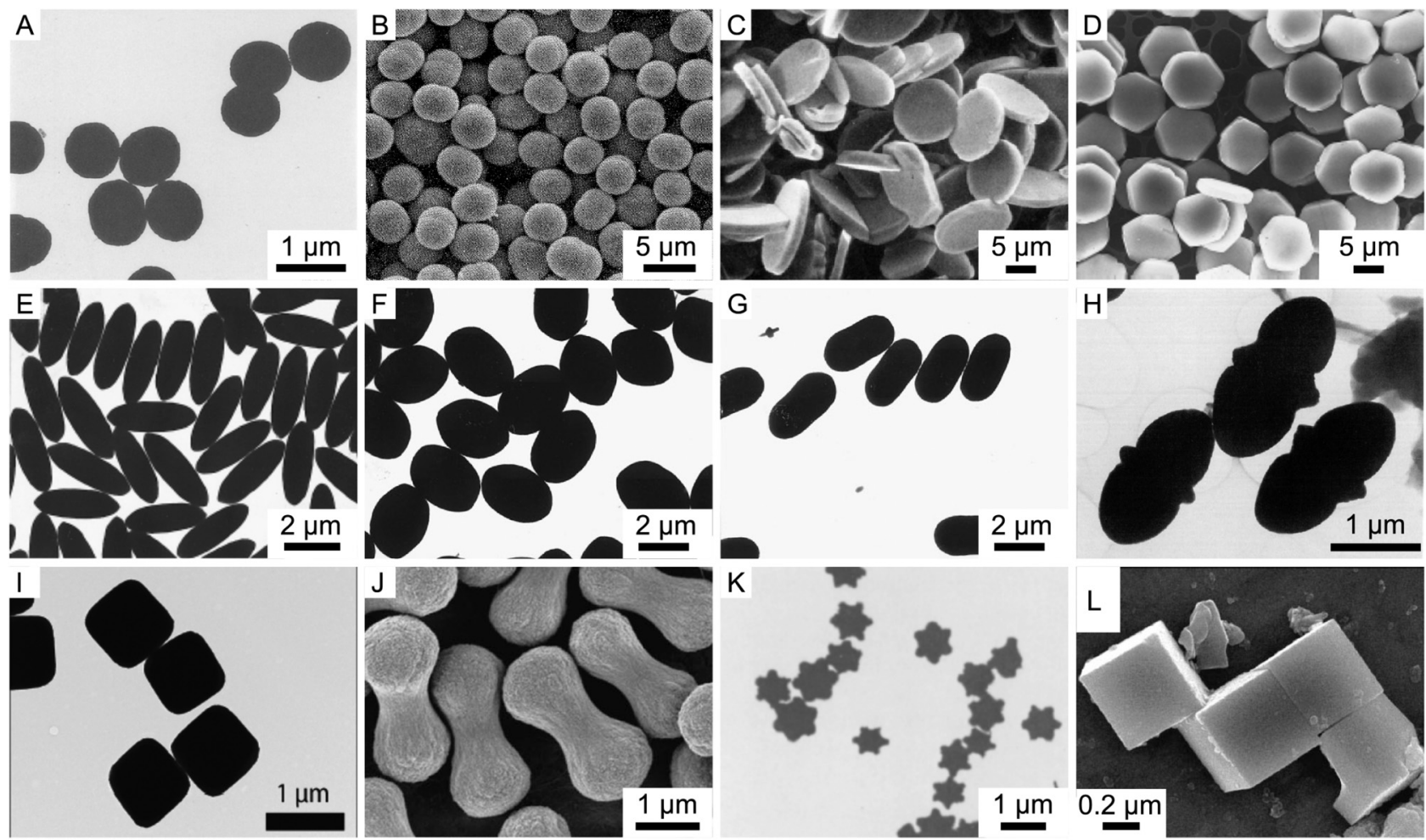

Fig. 1 The different shapes of uniform micron-sized hematite particles. TEM and SEM micrographs of monodisperse hematite particles with different shapes: (A) spheres, ${ }^{64}$ (B) double spheres, ${ }^{47}$ (C) round platelets, ${ }^{62}$ (D) hexagonal platelets, ${ }^{65}$ (E-G) ellipsoids, ${ }^{65,60}$ (H) double ellipsoid, ${ }^{32}$ (I) cubic, ${ }^{12}$ (J) peanut, ${ }^{10}(\mathrm{~K})$ bipyramid $^{1}$ and (L) single crystal cubes. ${ }^{66}$ Panel (A) reprinted by permission from Springer Nature, K. Kandori, S. Tamura and T. Ishikawa, Colloid Polym. Sci., 1994, 272, 812-819. Copyright 1994. Panel (B) adapted with permission from S. Hamada, S. Niizeki and Y. Kudo, Bull. Chem. Soc. Jpn., 1986, 59, 3443-3450 - The Chemical Society of Japan. Panel (C) adapted with permission from E. Matijevic, Acc. Chem. Res., 1981, 14, 22-29. Copyright 1981 American Chemical Society. Panel (D and E) reprinted from T. Sugimoto and Y. Wang, J. Colloid Interface Sci., 1998, 207, 137-149, Copyright 1998, with permission from Elsevier. Panel (F and G) reprinted from T. Sugimoto, H. Itoh and T. Mochida, J. Colloid Interface Sci., 1998, 205, 42-52, Copyright 1998 with permission from Elsevier. Panel (H) reprinted from J. K. Bailey, C. J. Brinker and M. L. Mecartney, J. Colloid Interface Sci., 1993, 157, 1-13, Copyright 1993 with permission from Elsevier. Panel (I) is adapted from L. Rossi, J. G. Donaldson, J.-M. Meijer, A. V. Petukhov, D. Kleckner, S. S. Kantorovich, W. T. M. Irvine, A. P. Philipse and S. Sacanna, Soft Matter, 2018, 14, 1080-1087 - Published by The Royal Society of Chemistry. Panel (J) is adapted with permission from S. H. Lee and C. M. Liddel, Small, 2009, 5(17), 1957-1962. Copyright 2009 Wiley-VCH. Panel (K) reprinted from E. Matijević and P. Scheiner, J. Colloid Interface Sci., 1978, 63, 509-524, Copyright 1978, with permission from Elsevier. Panel (L) adapted with permission from B. Jia and L. Gao, Cryst. Growth Des., 2008, 8, 1372-1376. Copyright 2008 American Chemical Society. 
Table 1 Non exhaustive overview of the different shapes in which uniform micron-sized hematite particle can be obtained and their synthesis conditions

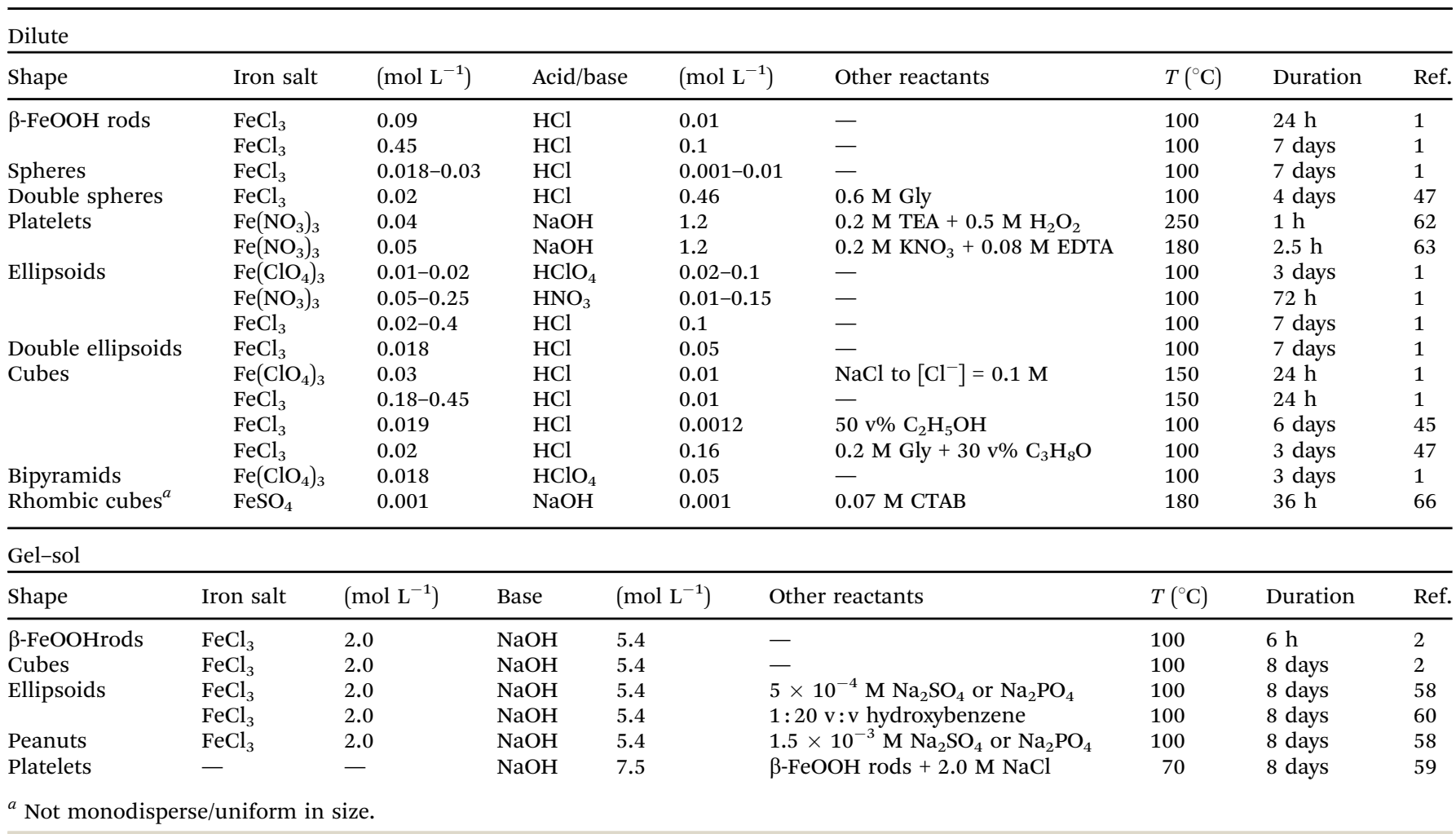

of control relates to the adsorption of different additives onto specific faces of the hematite crystals, thereby retarding the growth of these faces. The cubic particles are obtained because the chloride ions absorb onto the $\{012\}$ face. ${ }^{67}$ Ellipsoidal or peanut shaped particles are obtained with either sulphate or phosphate because both absorb onto the face parallel to the c-axis of hematite. ${ }^{65,68}$ Finally, hexagonal platelets are obtained as the hydroxide ions absorb onto the $\{001\}$ face of hematite. ${ }^{59}$ Besides these anions, organic additives introduced into the gel-sol method lead to the formation of different ellipsoidal shapes. ${ }^{60}$

For the forced hydrolysis method, the role of the anions has been more difficult to establish because the shape and size outcome, as explained before, also depends on the exact concentrations, temperature and ageing time. Some shape regimes were established by Matijević and others by making extensive systematic overviews of different iron salt concentrations versus acid or other additive concentrations, as well as the effect of ageing time. ${ }^{1,46}$ The overview provided in Table 1 shows that the range of shapes obtained with forced hydrolysis is slightly more abundant than the gel-sol method. It is interesting to note, however, that the peanut shapes were never obtained with the forced hydrolysis method. The table shows that ellipsoids can be obtained with $\mathrm{Fe}\left(\mathrm{NO}_{3}\right)_{3}, \mathrm{Fe}\left(\mathrm{ClO}_{4}\right)_{3}$ and $\mathrm{FeCl}_{3}$, but that spheres and bipyramidal shapes can also form using perchloride salt, while spheres, double ellipsoids and cubes can be obtained with the chloride. ${ }^{1}$ Other studies have shown that organic and inorganic sulphates will promote the formation of different ellipsoid shapes, ${ }^{33,58}$ while glycine leads to the formation of double spheres ${ }^{47}$ and alcohols will change spheres into cubic shapes. $^{46}$

Interestingly, when employing $\mathrm{FeSO}_{4}$ as a starting salt in the forced hydrolysis method with different additives, other unique shapes can be obtained. With urotropine or cetyltrimethylammonium bromide (CTAB) ${ }^{66,69}$ the resulting shapes are rhombic cubes with a unique single crystal nature as shown in Fig. 1L. In addition, when $\mathrm{FeSO}_{4}$ is combined with $\mathrm{CO}\left(\mathrm{NH}_{2}\right)_{2}$ in wateralcohol mixtures, hematite nests and chesnut buds can be obtained. ${ }^{70}$ This is surprising, as many of the other hematite particles discussed so far typically possess a particular needlelike interior as discussed in Section 3.2. However, we do note that the resulting particles are rather polydisperse in size, in marked contrast with the particles obtained from $\mathrm{FeCl}_{3}$.

Typically, the studies revealing the influence of different additives, although sometimes accidental, only explored a limited sets of synthesis conditions. Therefore, it would be interesting to investigate different concentration regimes in a similar systematic manner as in the works of Matijevic to understand whether monodisperse particles with new unique shapes can be obtained.

\subsection{Formation mechanism}

In this section we will focus on the formation mechanism for hematite particles obtained from $\mathrm{FeCl}_{3}$ solutions, as this is the most commonly employed salt in both the forced hydrolysis and gel-sol method. The growth process of hematite particles 
occurs via a two-step transformation from the hydrolyzed iron species to akaganéite rods and from the akaganéite rods to hematite particles. ${ }^{1,2}$ The three stages can be described by the following reactions:

(1) precipitation of $\beta$-FeOOH via hydrolysis of iron

$$
\mathrm{Fe}^{3+}+3 \mathrm{OH}^{-} \rightarrow \mathrm{Fe}(\mathrm{OH})_{3} \rightarrow \beta-\mathrm{FeOOH}+\mathrm{H}_{2} \mathrm{O}
$$

(2) dissolution of $\beta$-FeOOH

$$
\beta-\mathrm{FeOOH}+\mathrm{H}_{2} \mathrm{O}+3 \mathrm{H}^{+} \rightarrow \mathrm{Fe}^{3+}+3 \mathrm{H}_{2} \mathrm{O}
$$

(3) precipitation of $\alpha-\mathrm{Fe}_{2} \mathrm{O}_{3}$ :

$$
2 \mathrm{Fe}^{3+}+3 \mathrm{H}_{2} \mathrm{O} \rightarrow \alpha-\mathrm{Fe}_{2} \mathrm{O}_{3}+6 \mathrm{H}^{+}
$$

The total transformation process can take anywhere between 8 hours to 20 days, depending on the exact synthesis conditions. During this whole process, aggregation of the hematite particles is prevented by the electric double layer repulsion between the particles at low ionic strength or by encapsulation of the particles in the gel network in the gel-sol method. Due to the step-wise process, the nucleation and growth stages of the hematite particles are separated and lead to the formation of uniform sizes and shapes.

A schematic overview of the process is shown in Fig. 2. After the hydrolysis of iron(III), induced by heating the dilute solutions or by addition of the alkaline solution in the gel-sol system, the rapid formation of the akaganéite needles occurs.

During the akaganéite formation, also the nucleation of small hematite precursors occurs but it is quickly halted by the sharp drop in $\mathrm{pH}$ accompanying the akaganéite formation. ${ }^{46,47,67}$ This process was confirmed by studies showing that changes in the initial $\mathrm{pH}$ lead to a change in the number of nuclei formed. ${ }^{67}$ Next, the hematite particles start to grow via a dissolution-recrystallization process where iron species dissolve from the akaganéite needles and redeposit on the small hematite nuclei. It has been shown that in a reaction mixture the increase in solid content of hematite is inversely related to the decrease of solid content of the akaganéite. ${ }^{47,65}$ At the same time, the concentration of iron species in the solution phase has been found to remain constant around the solubility level of the akaganéite rods, while the rods also show Ostwald ripening. ${ }^{68}$ The rods in this way provide a continuous source for monomeric ferric species that directly deposit onto the growing hematite particle surface. The reaction rate of the ferric precursor complexes at the surface of growing hematite has been shown to be the rate-determining step of particle growth in seededgrowth experiments. ${ }^{65,71}$ The deposition of the ferric complexes species onto the hematite nuclei surface is not homogeneous and leads to the formation of small subcrystals. In addition, the reaction speed at the surface is regulated by the absorbance of anions onto the different hematite crystal planes and thus leads to the anisotropic growth of these subcrystals and finally the full particle shape. ${ }^{65}$ Simulations have shown that inhibited surface growth perpendicular to $c$-axis and flexibility in the angle of attachment of the hematite subcrystals can explain the peanut shape formation. ${ }^{72}$

For the growth of hematite particles from dilute solutions of $\mathrm{FeCl}_{3}$ the dissolution-recrystallization mechanism just described has been debated. Already as early as the 80s, but also more recently, studies propose different growth mechanisms, including oriented attachment, aggregation of akaganéite needles and direct phase transformations into hematite. ${ }^{32,48,73,74}$ The main claims are often based on TEM investigations, which is not an in situ method, and to obtain these images several manipulations need to be performed on the particle suspensions, such as cooling, redispersion and centrifugation. In that sense, cryo-TEM is a better technique because it allows direct imaging of the particle in the solvent. ${ }^{74}$ However, also in this case, the

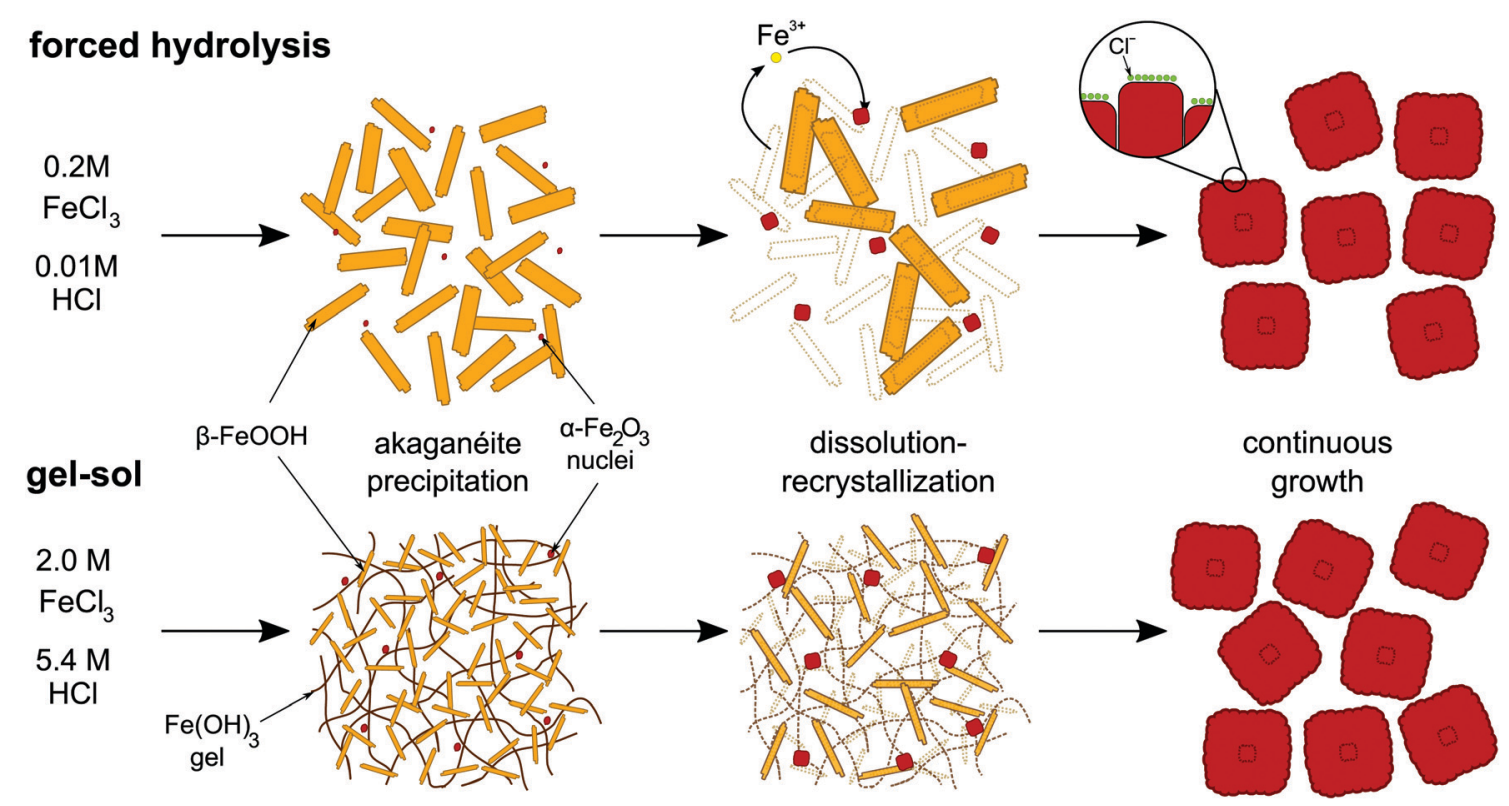

Fig. 2 Schematic representation of the formation mechanism of hematite cubes in the forced hydrolysis and gel-sol methods. 
reaction has to be quenched and samples disturbed before the particles can be imaged in a frozen liquid. In this respect, the detailed and extensive analysis with numerous techniques as well as quantitative seeded growth experiments performed by Sugimoto, ${ }^{71}$ motivated by the earlier debate, have shown rather conclusively that the dissolution-recrystallization mechanism occurs for hematite particles from dilute solutions. In addition, the other proposed mechanisms cannot explain the abundance of shapes obtained by a small change in acid concentration or the presence of additives, whereas a growth mechanism controlled by the surface reaction of monomeric ferric species does. However, the newer techniques available to us today, such as liquid TEM, might shed new light on the hematite particle growth mechanism.

\subsection{Preparation control}

While both synthesis methods appear relatively easy to reproduce, there are several practical aspects that need to be controlled properly when performing the synthesis, especially in the case of the gel-sol method. For both methods, one of the first challenges one encounters during the synthesis is that iron(III) salts are hygroscopic. This fact complicates the exact weighing of the desired amounts of salts in normal laboratory conditions and hence makes determining the exact concentration of iron difficult, which is a crucial parameter as the following shows. To solve this problem, a controlled environment can be employed, for example by using a glove box, or the exact $\left[\mathrm{Fe}^{3+}\right]$ concentration can be determined via spectroscopic means prior to the synthesis.

In the forced hydrolysis method, as mentioned before, the size and shape of the particles are controlled by:

- nature and concentration of iron(III)salt.

- concentration of acid and/or base.

- nature and concentration of additives.

- temperature and ageing time.

Thus, the desired particles can be obtained by mixing the desired concentration of an iron(III) solution with the desired concentrations of acid/base/additive, heating to the correct temperature and ageing for a specific time. Beside carefully controlling and checking the concentrations of the solutions, the outcome is not sensitive to how the solutions are mixed and small disturbances during the formation process also do not seem to be an issue.

For the gel-sol method, however, the synthesis conditions are much more crucial. The reason is that the exact outcome depends very precisely on how the first mixing step is performed since during this step both the formation of the $\mathrm{Fe}(\mathrm{OH})_{3}$ gel as well as the nucleation of precursor hematite particles occur. To obtain hematite particles with the desired size and a narrow size distribution using the gel-sol method, only a single burst of nuclei should be triggered and the following practical preparation aspects should be controlled: ${ }^{67,68,75}$

- excess $\left[\mathrm{Fe}^{3+}\right]$ concentration.

- addition speed of the $\mathrm{NaOH}$ solution.

- the agitation speed of the mixture.

- temperature of the solutions.
- preparation scale.

For the first item, the reason is that the final mean particle size is determined by the nominal excess concentration of $\left[\mathrm{Fe}^{3+}\right]$ with respect to the $\left[\mathrm{OH}^{-}\right]$concentration. An excess concentration of $0.10 \mathrm{M}$ yields micron size particles while a reduction to $0.01 \mathrm{M}$ yields particles of a few hundred nanometers. ${ }^{2}$ Thus, the initial iron and hydroxide concentration should be carefully determined. Regarding the other aspects, controlling the first mixing step was shown to influence the nucleation event in different ways. ${ }^{75}$ Specifically, fast addition of the $\mathrm{NaOH}$ solution leads to faster nucleation and smaller final particle size, while slow addition results in less nuclei, larger final particle size and eventually akaganéite rods as a side final product. ${ }^{68}$ In addition, agitation of the gel during the phase transformation process will lead to several separate nucleation events and a large distribution in particle sizes. ${ }^{75}$ Moreover, increasing the temperature of the iron and $\mathrm{NaOH}$ solutions (which occurs naturally as the reaction is exothermic) will increase nucleation speed and hence decrease the size of the particles. Finally, the preparation scale of the synthesis should be kept constant if a specific parameter is changed to, for instance, obtain a different particle size. The reason is that changing the scale at which the synthesis is performed will inevitably influence the other control parameters and thus change the synthesis outcome.

So, for those readers that have tried the gel-sol synthesis themselves and were puzzled about a difference in size outcome, such as the authors of this review have been, our suggestion is to work towards controlling every aspect of the preparation procedure. A clear giveaway for a synthesis gone bad is the color of the solutions and suspensions. The final suspension of hematite particles should possess a bright red almost blood-like color (as the name suggests). Akaganéite rods, however, possess a yellow color and hence their presence can be clearly spotted. In addition, the hematite color will also give away if the correct excess concentration of iron was employed. For instance, depending on their size, the color of cubic hematite particles changes from bright orange, via blood red to reddish purple for an edge length of $\sim 300 \mathrm{~nm}$ to $\sim 500 \mathrm{~nm}$ to $\sim 1 \mu \mathrm{m}$, respectively. A clear example can be found in ref. 11 .

\section{Internal structure and physico-chemical properties of micron-sized hematite particles}

The large number of applications of hematite microparticles in the soft matter field are attributable to their unique physicochemical properties. In this section we will touch upon the most relevant properties that can directly be utilized in soft matter applications.

\subsection{Surface chemistry and colloidal stability}

Hematite particles are stabilized by repulsion of their electrostatic double layers caused by their surface charge. The surface 
charge characteristics of the hematite particles are dependent on the solution $\mathrm{pH}$ as shown by electrokinetic mobility measurements with a zero point charge (z.p.c.) around 6, with a positive surface charge below and a negative surface charge above the z.p.c. Practically, this means that in deionized water with a typical $\mathrm{pH}$ of $\sim 5.5$, the hematite particles will no longer be charge stabilized and will therefore aggregate. This is in contrast to, for instance, silica colloids, which are fully charge stabilized in deionized water since its z.p.c. is around pH 2 . For the hematite shapes obtained with the forced hydrolysis synthesis it has been found that the shape has a limited influence on the z.p.c. Most shapes have the approximate value of 5.9 with the exception of cubes and ellipsoids for which a z.p.c. of $\sim 6.6$ was reported, see ref. 1 for the full $\mathrm{pH}$ dependence. The differences in z.p.c. between shapes can be attributed to the different shape controlling anions (see Section 2.2) that get incorporated into the interior of the different particles. In case of cubes these are chloride ions while for ellipsoids these can be sulphate or phosphate ions. Noteworthy is that for spherical hematite particles the shape controlling chloride ions were found to be slowly released from the particles interior over time. These loosely bound chloride ions were only completely removed from the surface after 35 washings steps, thereby shifting the z.p.c. from $\sim 5.9$ to $\sim 7.1 .^{76}$ It was also noted that ageing of the particles may result in chloride ions diffusing from the interior to the surface again, which can affect their electrokinetic mobility. It is therefore very important to carefully control the $\mathrm{pH}$ as well as the ionic strength of solutions when performing experiments with hematite particles. The important role of the z.p.c. has been shown in two recent studies on the stability of pickering emulsions of hematite particles with different shapes. ${ }^{77,78}$

\subsection{Internal structure}

The internal structure of hematite microparticles is quite unusual, especially when accounting for their magnetic properties. Early analysis of the internal structure of cubic, ${ }^{79}$ peanut-like ${ }^{80}$ and plate-like ${ }^{81}$ hematite were done in the group of Sugimoto who is also responsible for pioneering work on the synthesis of the same particles. In their work, thin slices of particles were cut using an ultramicrotome and then analyzed by high-resolution electron microscopy (HREM). For hematite platelets, HREM analysis revealed particles with a single crystalline structure, accompanied by the occasional crystal defect. ${ }^{81}$ Cubic and peanut-like particles, however, showed the presence of elongated subcrystals with a preferential alignment depending on the particle type. For cubic particles (Fig. 3A), the subcrystals seem to radially develop from the center of the particles in all directions towards the surface, with a preference along the $c$-axis of the cube; for peanut-like particles, ${ }^{80}$ the subcrystals are radially oriented from the $c$-axis of the particle which in this case corresponds to the long axis of revolution. A more recent work by Lee et al. ${ }^{10}$ confirms, using the same characterization technique, the findings for internal structure of peanut-like particles (Fig. 3C). Additional studies on ellipsoidal/spindle-like particles grown in the presence or organic additives ${ }^{60}$ have also
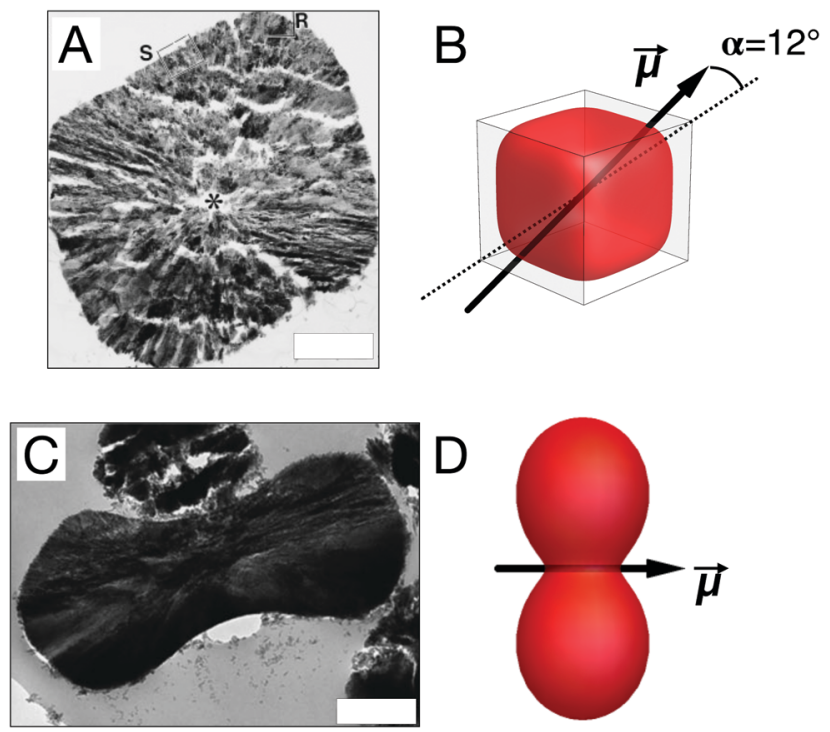

Fig. 3 Internal structure and magnetic properties. Thin sections of a cubic $^{79}(\mathrm{~A})$ and peanut ${ }^{10}$ (C) shaped hematite particle imaged with Transmission Electron Microscopy (TEM) showing their internal structure. Models of cubic $^{12}$ (B) and peanut ${ }^{10}$ (D) shaped hematite particles with their respective dipole moment orientation. Scale bars are $500 \mathrm{~nm}$. Panel (A) is adapted with permission from G. Park, D. Shindo, Y. Waseda and T. Sugimoto, J. Colloid Interface Sci., 1996, 177, 198-207. Copyright 1996 Elsevier. Panel (C) is adapted with permission from S. H. Lee and C. M. Liddel, Small, 2009, 5(17), 1957-1962. Copyright 2009 Wiley-VCH.

shown the presence of subcrystals. While these works have provided valuable insights into the internal structure of micron-sized hematite particles, a deeper understanding on the relationship between internal structure and magnetic properties is currently still lacking.

\subsection{Magnetic properties}

One of the most fascinating aspects characterizing hematite microparticles relates to their magnetic properties. Of all the oxides and materials commonly used for the preparation of magnetic colloids, ${ }^{82}$ hematite is the only one which allows to naturally obtain permanently magnetized particles with size in the order of $1 \mu \mathrm{m}$ and above. The reason is that, while its spontaneous magnetization is lower than that of commonly used magnetic materials, its domain size is of the order of $15 \mu \mathrm{m} .{ }^{8}$ Although it has always been clear that hematite was a magnetic material, it is only in the late 1950's when hematite was first identified as a canted antiferromagnet, ${ }^{83}$ in which the canting of the magnetic spins is caused by the so-called Dzyaloshinskii-Moriya interaction..$^{83,84}$ In bulk crystalline hematite, the canting of the spins is temperature dependent. Below the so called Morin temperature ${ }^{85}\left(T_{\mathrm{M}} \approx 250 \mathrm{~K}\right)$ the spins are aligned with the [111] axis giving rise to a pure antiferromagnetic arrangement. Above the Morin temperature, the spins flip on the (111) plane in which they experience the canting. The extent of the spin canting has been investigated by neutron diffraction measurements, and during the years, a variety of tilt angles have been reported: $7^{\circ},{ }^{86} 5^{\circ},,^{87} 15^{\circ} \pm 10^{\circ}$ 
for polycrystalline hematite. ${ }^{88}$ For colloidal hematite cubes, it was recently reported that the overall dipole moment is located at a $12^{\circ}$ angle from one of the internal diagonals of the cubes ${ }^{12}$ as shown in the model of Fig. 3B. However, due to the symmetric nature of the cubic shape and the peculiar internal structure of the particles, relating the overall dipole orientation to the spin canting in the bulk crystal structure is not straightforward. For hematite microparticles with an elongated shape and similar internal structure, such as ellipsoidal and peanutlike particles, the dipole moments has always been reported to be perpendicular to the long axis ${ }^{9,10}$ (see Fig. 3D), mostly due to their tendency to form linear dipolar chains in applied field. However, no specific measurements targeting the magnetic properties for elongated hematite microparticles have been reported to date. An extensive account on the magnetic properties of hematite can be found in the book by Morrish. ${ }^{7}$

Because of hematite's relatively low spontaneous magnetization $\left(M_{\mathrm{s}, \mathrm{h}} \approx 2.2 \times 10^{3} \mathrm{~A} \mathrm{~m}^{-1}\right)$ particles need to be relatively big to be able to produce interparticle interactions that are strong enough to promote magnetically driven self-assembly. For instance, a cubic hematite particle with an edge length of $1 \mu \mathrm{M}$ has an approximate magnetic moment of $\mu_{\mathrm{h}} \approx 2.2 \times$ $10^{-15} \mathrm{~A} \mathrm{~m}^{2}$. For comparison, a particle with the same magnetic moment from magnetite, whose spontaneous magnetization is two order of magnitudes larger than that of hematite $\left(M_{\mathrm{s}, \mathrm{h}} \approx\right.$ $4.8 \times 10^{5} \mathrm{~A} \mathrm{~m}^{-1}$ ), one would need a cube with edge length of approximately $160 \mathrm{~nm}$, which coincidentally represents the experimental upper limit size for obtaining single domain cubic magnetite particles. ${ }^{89}$ This is one of the reasons why, while some studies have been done on hematite nanoparticles, ${ }^{35,38,90,91}$ we focus here our attention on micron-sized hematite colloids, which magnetic interactions are strong enough to create systems that can spontaneously self-assemble and respond to relatively weak magnetic fields.

\subsection{Photocatalytic properties}

Hematite is a well-known semiconductor. When a semiconductor is hit by light with the appropriate wavelength, it promotes the formation of an electron $\left(\mathrm{e}^{-}\right)$/hole $\left(\mathrm{h}^{+}\right)$pair. This charge separation can then be used to catalyze redox reactions on the particles' surface, in a process commonly called heterogeneous photocatalysis. While there is still some debate about it, the most commonly accepted bandgap value for hematite is of the order of 1.9-2.2 $\mathrm{eV}^{92,93}$ depending on the synthesis method. Because of this narrow bandgap, hematite is ideally suited for visible light absorption compared to other catalysts. This is one of the most attractive catalytic properties of hematite, since it allows to directly harness solar light. This is, for instance, not possible with one of the most commonly used catalyst, titanium oxide, whose large bandgap (3.0-3.2 eV) enables absorption just in the UV range. ${ }^{94}$

In addition, hematite is a cheap and widely available material, is non-toxic, chemically stable and magnetic. One limitation, typical of iron oxide semiconductors, is the fast $\mathrm{e}^{-} / \mathrm{h}^{+}$ recombination rate which neutralizes the charge separation and, as a consequence, decreases the photoactivity of the hematite. Another limitation is the low hole mobility at room temperature which is in the order of $10^{-2} \mathrm{~cm}^{2}\left(\mathrm{~V}^{-1} \mathrm{~s}^{-1}\right)^{95}$ and poor hole diffusion length $(2-4 \mathrm{~nm}) .{ }^{96}$ Despite these drawbacks, the narrow bandgap makes hematite a promising material for many applications including photoanodes in photoelectrochemical water splitting. ${ }^{97-100}$ Furthermore, it is possible to enhance the photocatalytic activity of hematite ${ }^{100,101}$ for instance, by changing its morphology, ${ }^{102}$ through doping ${ }^{103}$ or coupling with a cocatalyst, ${ }^{104}$ something that can in principle be implemented also in the case of the well-defined monodisperse systems that we are describing here. Due to their photocatalytic properties, which are triggered by the illumination of the sample with light with an energy higher than the bandgap, hematite microparticles can be used to catalyze the decomposition of "fuels" such as hydrogen peroxide. Decomposition of a fuel near the surface of the particles creates a chemical gradient which in turn can trigger the osmotic self-propulsion of the particles, ${ }^{27}$ making the colloids "active". This is a very intriguing application for hematite microparticles that will be described later in Section 4.3.

\section{Current applications}

There is currently an abundance of applications in colloid and soft matter science that rely on the versatility and unique properties of hematite microparticles. In this section we will summarize the most important and promising applications which span from synthesis of model systems with various shapes and magnetic functionalities to study of in- and outof-equilibrium assemblies.

\subsection{Templates for complex colloids}

As we have seen in Section 2, the synthesis of hematite particles is quite versatile; uniform particles of different shapes can be controllably prepared in high yields and in various size ranges. These characteristics combined with their magnetic properties, make hematite microparticles ideal candidates for the design of more complex colloidal particles. One of the most straightforward application for hematite particle is shape-templating. The variety of shapes attainable with hematite particles, a collection of which is showing in Fig. 1, allows their use to mold other, usually amorphous, materials into shapes that would not be easily, if at all, accessible through conventional synthesis methods. Fig. 4 shows silica cubes (panels A, B) and ellipsoids (panel C) prepared by first growing amorphous silica on the surface of the hematite microparticles and then dissolving the hematite core in acidic environment through the porous silica shells ${ }^{22}$ as shown in the schematic of Fig. 4A. Novel cubic silica particles prepared with this technique were then used to self-assemble simple cubic crystals in the presence of attractive depletion forces, ${ }^{22}$ an example of which is shown in Fig. 4A. Growing silica layers of various thickness yields the ability to change the effective roundness of the cubes, allowing the study of shape-dependent assembly under various conditions. $^{23-26}$ Silica peanut-like particles templated from 


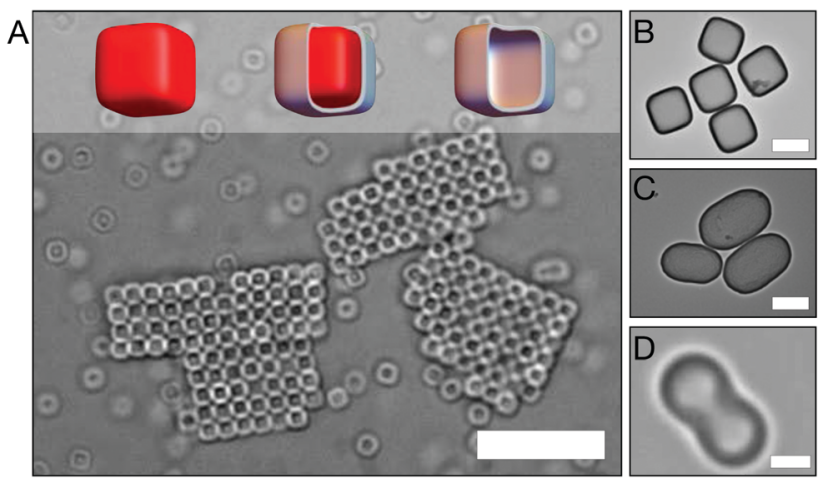

\section{E}
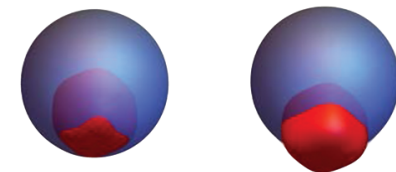

DEWETTING
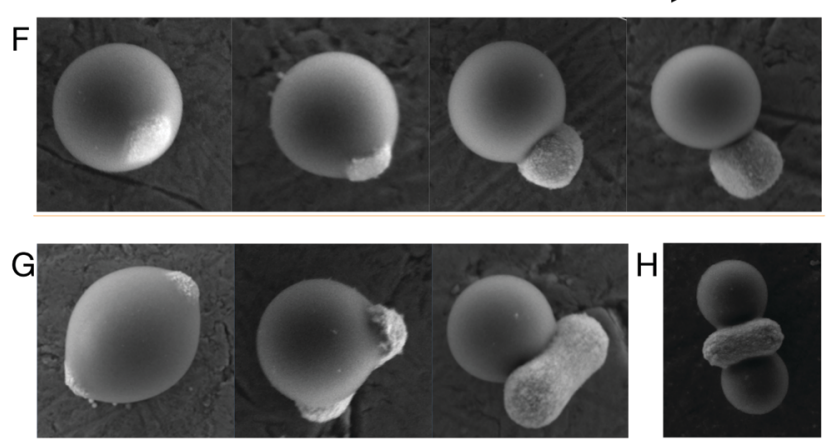

Fig. 4 Complex colloids from hematite microparticles. Silica cubic particles are prepared by depositing amorphous silica on the surface of hematite microparticles and subsequently dissolving the hematite cores as schematically shown in the models of panel (A). The silica cubic particles are further applied for self-assembly studies of attractive cubic particles. ${ }^{22,23}$ An example of cristallite formed by cubic silica particles is shown in the optical microscope image of panel (A). Transmission electron microscopy images of silica cubes (B) and ellipsoids (C) prepared with the same template technique. (D) Optical microscope image of a polystyrene peanut-like particle. ${ }^{107}$ (E) Schematic representation of the dewetting process that allows the preparation of composite TPM-hematite particles with various degree of anisotropy. ${ }^{109}$ The same procedure is visualized using scanning electron microscopy for cubic (F) and peanut-like (G) hematite particles. ${ }^{109}(\mathrm{H})$ Performing multiple growth and dewetting processes leads to the formation of more complex particles. Scale bars: $10 \mu \mathrm{m}$ in (A), $1 \mu \mathrm{m}$ in (B), (C), (D). Panel (D) is reprinted with permission from T. Hueckel, S. Sacanna, ACS Nano, 2018, 12, 3533-3540. Copyright 2018 American Chemical Society. Panels (F), (G) and $(H)$ are reprinted with permission from M. Youssef, T. Hueckel, G. Yi, S. Sacanna, Nat. Commun., 2016, 7(1), 12216.

hematite were prepared in a similar way by Lee et $a .^{21}$ These particles were then used to study their assembly into crystalline structures under confinement ${ }^{21}$ and the role of this crystal structure on dislocation propagation. ${ }^{105}$ A later work which summarizes and combines the preparation of hollow silica shells with cubic, ellipsoidal and peanut shapes can be found in ref. 106. Although, somehow, no reference to the earlier works is given. Polystyrene peanut-like particles via templating (Fig. 4D) were introduced in a more recent work by Hueckel and
Sacanna. ${ }^{107}$ Molding polystyrene into a peanut-shaped particles occur via a three steps process: first a hematite particle is decorated with small uncrosslinked sulfonated polystyrene spherical particles, then the polystyrene particles are melted on the surface of the hematite with tetrahydrofuran and finally the hematite core is dissolved in acidic environment. A similar templating technique was also employed for the preparation of cubic titania particles. ${ }^{108}$ To date, other available hematite shapes have yet to be explored for templating.

More complex colloidal particles can be prepared when hematite microparticles are combined with other nonmagnetic materials in various ways to create specifically responsive magnetic units. A very simple, yet powerful, example is the encapsulation of hematite cubic particles into (3-(methacryloxypropyl)trimethoxysilane, TPM) droplets. ${ }^{15}$ These particles are prepared by encapsulating the hematite microparticles into hydrolyzed TPM droplets via seeded growth. The amount of TPM deposited on the hematite determines the final size of the spherical droplet. Subsequent radical polymerization of the TPM oil allows hardening of the oil droplets to produce stable composite colloids (Fig. 4E, left model). A modification of this synthesis procedure was later presented to induce dewetting of the oil phase from the hematite surface ${ }^{109}$ before radical polymerization. The proposed method allows for morphological changes that effectively increase the degree of particle anisotropy as shown in the models of Fig. $4 \mathrm{E}$ and in real samples prepared with cubic (panel F) and peanut-like (panel G) hematite particles. This synthesis technique can be employed to prepare even more complex structures such as the one shown in Fig. $4 \mathrm{H}$, in which two TPM droplets have been nucleated on hematite peanuts. As we will see in Section 4.3, these particles can be further used to induce pre-programmed reversible assembly of non-linear structures and to model active and out-of-equilibrium systems.

Hematite cubic particles have also recently been used to create geometrically complex shapes by growth of silica rods selectively on each of the cube's faces. ${ }^{110}$ The silica rods are grown on the cubes from small water droplets that selectively nucleate on the cubes and subsequently promote silica growth. Depending on the initial water-oil concentration, the thickness of the rods can be varied to promote the formation of composite particles with different degrees of anisotropy. A very similar procedure has appeared later, ${ }^{111}$ even though no reference to the previous work ${ }^{110}$ was given, in which, in addition to growing silica rods on cubic particles, authors have extended the work to peanut- and disk-shaped hematite particles.

It is worth mentioning that templating can also be done using hematite nanoparticles. Many works, however, have focused on the use of spindle-shaped particles, ${ }^{91,112-117}$ which is the easiest and most common shape that can be prepared at the nanoscale.

\subsection{Colloidal assembly powered by magnetic interactions}

To achieve high levels of structural complexity in colloidal selfassembly, it is important that colloids possess specific and directional interactions. These can be obtained by using 
different means and techniques such as chemical $^{118-120}$ or physical $^{121,122}$ functionalizations. In recent years, however, it started to become clear that magnetic interactions could also play a pivotal role in controlling colloidal self-assembly. ${ }^{12,15,123-125}$ In this respect, hematite microparticles could be instrumental for the development of such colloidal building blocks due to their unique magnetic properties, which generally allow to achieve interparticle interaction energies that are well above the thermal energy $k_{\mathrm{B}} T$. Coming in many different shapes as described in Section 2, they provide a lot of options in terms of designing magnetic building blocks. So far, however, works which report using magnetic hematite microparticles as building units mostly focus on the simplest, and most commonly known shapes such as ellipsoidal, cubic and peanut-like. In the specific case of cubic hematite particles, many of these works were even published before the magnetic properties were thoroughly investigated and the direction of the dipole moment (see Fig. 3) was indisputably determined. $^{12}$

The first works that shows the magnetic self-assembly capability of hematite microparticles date back to the 1980s when Matijevic et al. reported optical microscopy observations of the agglomeration of hematite cubes, ${ }^{3,4}$ ellipsoids $^{4}$ and later platelets $^{63}$ in the Earth's magnetic field. These works remained somewhat unseen by the self-assembly community for some time, and only started collecting recognition in recent years, when hematite microparticles started to claim their role as magnetic building blocks. Studies of the 2D dipolar assembly of peanut-like hematite particles, with a dipole moment which is believed to lie parallel to the short particle axis (see Fig. 3C), have shown particles aligned in $2 \mathrm{D}$ zig-zag chains and large dense monolayers with structures governed by a balance between magnetic forces, surface charge and steric interactions. ${ }^{10}$ The collective field-induced dynamics of peanut-like hematite microparticles were explored, in a different study, using ultrasmall-angle X-ray photon correlation spectroscopy (USAXPCS). ${ }^{13}$ This work targeted the particles dynamics that originate when dipolar chains form upon application of an external magnetic field and reported different dynamics in directions perpendicular and parallel to the applied field. The structure of sedimented silica-coated hematite peanut-like particles were investigated using Small Angle X-ray Diffraction with microradian resolution $(\mu \mathrm{rad}-\mathrm{XRD})^{126}$ to understand the influence of an applied magnetic field in sedimenting samples. It was found that the structure of sedimented samples of magnetic peanutlike particles can be tuned by applying the magnetic field either during of after sedimentation. For ellipsoidal particles, the 2D assembly behavior of a small number of particle in static and oscillating magnetic fields was reported in the work by Martinez-Pedrero et al. ${ }^{9}$ The same group also reported chain and ring formation in 2D samples of the same particles, demonstrating that controlled deformation of the rings under applied magnetic fields, as shown in Fig. 5A, can be used to entrap cells. $^{127}$

The bulk assembly of hematite magnetic cubes was first studied in large sediments using $\mu \mathrm{rad}-\mathrm{XRD} .{ }^{11}$ In this work, beside shape and dipole-dipole interactions, surface charge

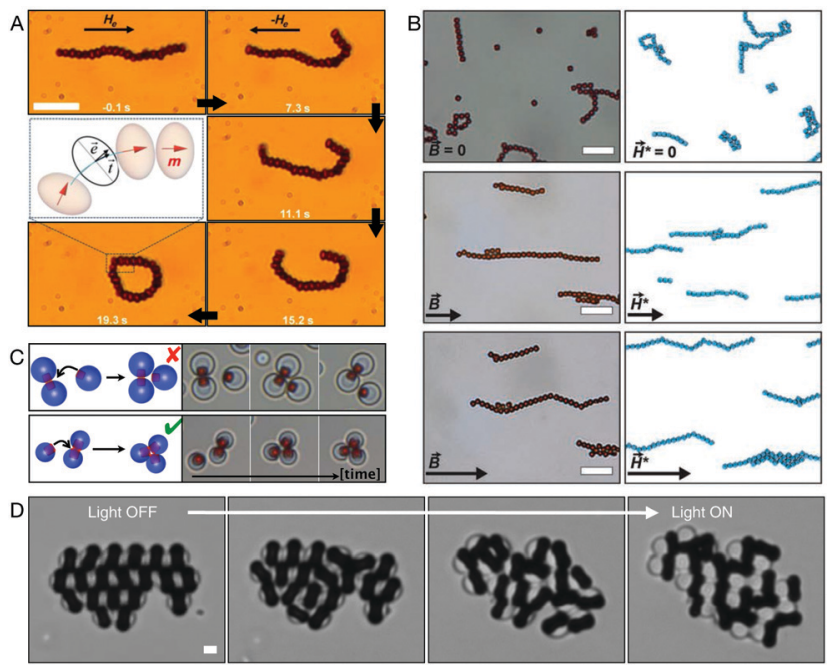

Fig. 5 Magnetic assembly. (A) Image sequence showing ellipsoidal hematite particles transitioning from a linear chain aligned with the Earth's magnetic field to a closed ring. Scale bar is $10 \mu \mathrm{m}$. Image adapted from ref. 127. (B) Experimental observations (left) and computer simulations (right) of hematite cubes assemblying in quasi-2D in zero-field environment (top), weak applied field (middle) and high field (bottom). Image adapted from ref. 12. Scale bars are $10 \mu \mathrm{m}$. (C) The same hematite cubes encapsulated in polymeric spheres can be programmed to form only dimers (top) or trimers (bottom) by changing the size of the polymer sphere. Image adapted from ref. 15. (D) A small crystallite composed of "shape-shifting" particles of the type shown in panel (G) of Fig. 4 are reconfigured using a light stimulus that changes the particles' anisotropy. Image adapted from ref. 109. Panel (A) is reprinted with permission from $\mathrm{F}$. Martinez-Pedrero, A. Cebers, and P. Tierno, Phys. Rev. Appl., 2016, 6, 034002. Copyright 2016 by the American Physical Society. Panel (B) is adapted from L. Rossi, J. G. Donaldson, J. Meijer, A. V. Petukhov, D. Kleckner, S. S. Kantorovich, W. T. M. Irvine, A. P. Philipse and S. Sacanna, Soft Matter, 2018, 14, 1080 - Published by The Royal Society of Chemistry. Panel $(C)$ is reprinted with permission from S. Sacanna, L. Rossi and D. J. Pine, J. Am. Chem. Soc., 2012, 134(14), 6112-6115. Copyright 2012 American Chemical Society. Panel (D) is reprinted with permission from $M$. Youssef, T. Hueckel, G. Yi, S. Sacanna, Nat. Commun., 2016, 7(1),12216.

was used as an assembly parameter. The alignment of cube faces due to osmotic pressure or dipolar alignment to an external magnetic field resulted in different crystal lattices. In the work that unraveled the orientation of the dipole moment in hematite cubes, ${ }^{12}$ the authors also documented the cooperation between particle shape and dipole moment orientation on the self-organization of the particles in quasi-2D samples in zero and applied magnetic fields. By means of experiments and computer simulations, the authors were able to quantitatively determine the contributions given by particle shape and magnetic properties and showed that even a slight variation in the dipole moment orientation lead to different assemblies as shown in Fig. 5B. In zero-field environment, it was found that hematite cubes form rings and chain-like structures resembling dipolar structures in magnetic spheres. In applied field, depending on the field strength, a transition between two different structures was observed: linear chains in weak fields, and kinked chains in stronger fields. This peculiar behavior is driven by competing anisotropic interactions between the 
particle shape and the internal orientation of the dipole moment.

The same magnetic cubes were also used to prepare spherical particles with an off-centered dipole moment to obtain more complex magnetic building blocks, ${ }^{15}$ as shown in the leftmost images of Fig. $4 \mathrm{E}$ and F. Since the hematite particle, and hence the dipole moment, is embedded in the polymeric sphere in an off-centered fashion, the resulting composite particles are good models of colloidal spheres with a single magnetic patch. ${ }^{128,129}$ By changing the size of the encapsulating polymeric sphere, particles can be pre-programmed to selectively form dimers or trimers, as shown in in the top and bottom panels of Fig. 5C, respectively. The dewetting process induced by light of composite hematite-TPM particles presented in Section 4.1 and shown in Fig. $4 \mathrm{E}, \mathrm{F}$ and $\mathrm{G}$ has been used to induce morphological changes in in small crystallites. Fig. 5D shows the reconfiguration of a twenty-particle crystal prepared with peanut-like hematite encapsulated in a spherical TPM matrix (Fig. $4 \mathrm{G}$ ) from a uniform crystal to a striped structure. ${ }^{109}$

Again it is noteworthy that for the hematite spindles the influence of magnetic fields on the phase behaviour and particle alignment for different composite systems and at high concentrations has been explored in detail by several groups. ${ }^{130-137}$

\subsection{Active colloids and out-of-equilibrium phenomena}

Active matter has recently become the focus of many research groups, touching several disciplines in soft matter, including physics, biology, chemistry and engineering. The reason for the growing interest in active matter is not only of fundamental relevance. Research in this area offer unique opportunities to engineer materials with specific functionalities that cannot be obtained in passive systems. Hematite microparticles are particularly suited for these kind of applications due to their intrinsic properties that have been presented in previous sections: high production yields, low production costs, magnetic and photocatalytic properties. For these reasons, recent years have seen the rise of a variety of attractive works involving hematite microparticles in the field of active colloids and outof-equilibrium assembly. Below we will introduce the most relevant applications of hematite microparticles in the field.

The first appearance of hematite as an active system is reported in the work of Palacci et al. ${ }^{27}$ There, colloidal swimmers were prepared by encapsulating hematite cubic particles into polymeric shells made of TPM, as shown in the leftmost model of Fig. 4E. In their work Palacci et al. have exploited the photocatalytic properties of hematite in order to propel the composite hematite-TPM particles. In brief, they have dispersed the composite particles in a basic solution containing hydrogen peroxide. When the sample is illuminated by light with wavelength $\lambda \approx 430-490 \mathrm{~nm}$, the particles propel due to a phoretic motion driven by a chemical gradient around the hematite surface whose face is exposed to the solvent through the TPM shell. This gradient is created when the hydrogen peroxide present in the solvent is decomposed into oxygen and water, a reaction that is catalyzed at the hematite surface illuminated by blue/violet light. The maximum 2D velocity of the active particles is approximately $15 \mu \mathrm{m} \mathrm{s}^{-1}$. At high concentrations, these propelling particles are able to form what the authors of the work called "living crystals"; two-dimensional crystal structures that dynamically form, disassemble and reform elsewhere. This behavior results from a balance between the self-propelling behavior and the attractive phoretic interactions that arise due to the light-induced chemical gradients. Due to the magnetic properties of the hematite microparticles, the crystals could be steered by applying external magnetic fields $(B \approx 1 \mathrm{mT})$. In a different work, ${ }^{28}$ the same group took advantage of both magnetic and photocatalytic properties of hematite to develop colloidal hematite dockers that can reach a cargo particle, dock and transport the cargo to a different location before releasing it. The particles used in this work have a peanut-like shape (see Fig. 3C and D). Both motion and docking are activated by the same osmotic/ phoretic mechanism presented above, while steering can be triggered by application of a weak magnetic field or by inducing the motion on a textured substrate. Another way in which the photocatalytic activity of hematite has been exploited in the soft matter field is for the design of self-powered microgears. ${ }^{30}$ Particles similar to those used in previous work, ${ }^{27}$ but with a higher degree of anisotropy ${ }^{109}$ (Fig. 4E and F), are first triggered by a focused laser to assemble into microgears with a certain chirality. These structures can then spin due to the selfpropelling feature of the particles forming the gear when exposed to blue light. These microgears have been shown to interact with each other and synchronize into superstructures as shown in Fig. 6A. The active hematite-TPM particles discussed so far have also been used to accelerate the annealing of monolayers composed of mostly non-active spherical particles. ${ }^{138}$ In a different work, Massana-Cid et al. ${ }^{139}$ have also shown how photoactive hematite ellipsoidal particles introduced in a system of passive silica spheres can aid the formation of large 2D gels and clusters, promoted by diffusiophoretic flows.

The same type of particles have also been shown to swim without consumption of chemical fuels. ${ }^{140}$ Moyses et al. have shown that light provided via a diverging laser beam induces propulsion through self-thermophoresis, which combined with an optically-induced torque, gives rise to complex trajectories.

Apart from their use as active particles, promoted by their photocatalytic properties, magnetic fields can also be used to actuate and drive particle motion. In ref. 141 peanut-shaped hematite particles were made to roll and wobble by application of spatially controlled magnetic field in 3D. By applying simultaneous rolling and wobbling movement, these particles were used to manipulate cells. Using this technique, it was shown that it is possible to organize cells in specific patterns on predefined PDMS substrates, while maintaining cell viability. Magnetic fields have also been used by Xie et al. ${ }^{19}$ to manipulate a collection of peanut-shaped hematite microparticles. In specific, the authors showed that using programmed alternating magnetic fields, they could control the formation of liquid, chain, vortex, and ribbon-like structures. The collective 

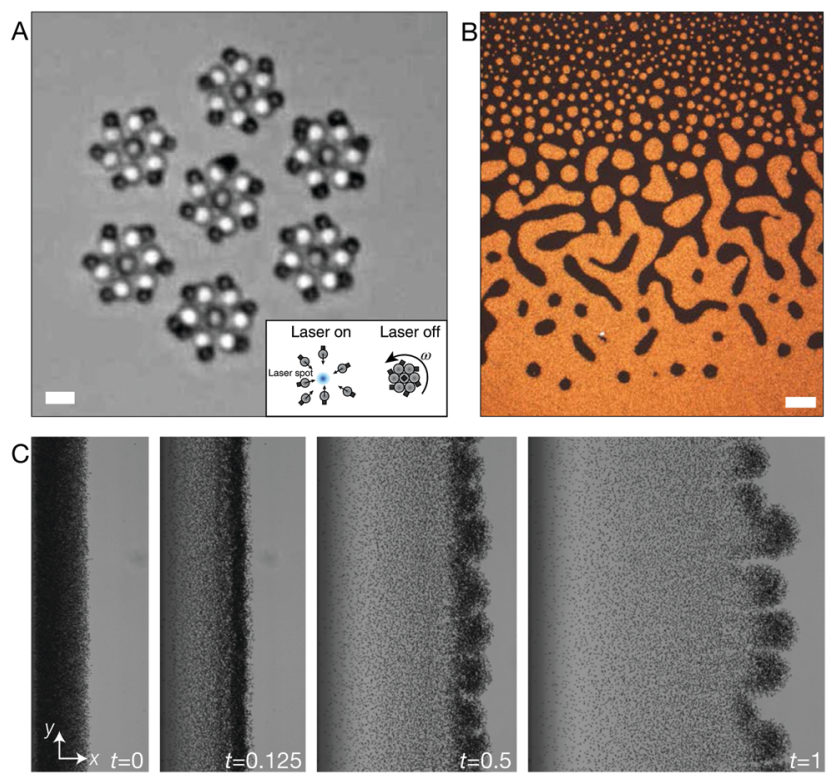

Fig. 6 Active colloids and out-of-equilibrium phenomena. (A) Optical microscope image of a collection of rotors that are first formed (as shown in the inset) and then confined by spatiotemporal light patterns. ${ }^{30}$ (B) Optical microscope image of a bulk suspension of hematite cubic particles after a few minutes of spinning by rotating magnetic fields. ${ }^{18}$ (C) Optical microscope image of the instability produced in time by rolling hematite-TPM composite particles. ${ }^{17}$ (A) Scale bar $2 \mu \mathrm{m}$ (B) scale bar $100 \mu \mathrm{m}$. Panel (A) is reprinted with permission from A. Aubret, M. Youssef, S. Sacanna, J. Palacci, Targeted assembly and synchronization of selfspinning microgears, Nat. Phys., 14(11), 1114-1118. Copyright 2018 Nature Publishing Group. Panel (B) is reprinted with permission from V. Soni, E. S. Bililign, S. Magkiriadou, S. Sacanna, D. Bartolo, M. J. Shelley, W. T. M. Irvine, The odd free surface flows of a colloidal chiral fluid, Nat. Phys., 15(11), 1188-1194. Copyright 2019 Nature Publishing Group. Panel (C) is reprinted with permission from M. Driscoll, B. Delmotte, M. Youssef, S. Sacanna, A. Donev, P. Chaikin, Unstable fronts and motile structures formed by microrollers, Nat. Phys., 13(4), 375-379. Copyright 2016 Nature Publishing Group.

magnetic manipulation of particles allowed to sequentially and controllably reconfigure the particles from one structure to the other effectively performing collective tasks such as passage through a narrow channel.

Hematite particles' intrinsic magnetism can also be used to promote other out-of-equilibrium phenomena. Soni et al. have recently reported the creation of a two-dimensional chiral liquid using spinning hematite cubes. ${ }^{18}$ This millimiter-scale cohesive chiral fluid, shown in Fig. 6B, was obtained by spinning a collection of $1.6 \mu \mathrm{m}$ hematite cubic particles with a magnetic field. Rotating magnetic fields were also used by Driscoll et al. to create and study the behavior of colloidal rollers. ${ }^{17}$ In this work, a collection of composite hematite-TPM particles are made to rotate and translate parallel to a glass wall by a rotating magnetic field with specific angular frequency. The authors report a novel fingering instability that create what they called 'critters' by pinching off from an unstable front, a phenomenon that is driven by hydrodynamic interparticle interactions alone. An image of the fingering instability is shown in Fig. 6C.

\section{Perspectives and conclusion}

The growing demand for smart, active, and controllable systems has brought a revolution in the way novel colloidal and soft matter systems are designed. In this respect, hematite microparticles have recently claimed a central role by providing unique attributes that have given scientists the ability of designing and developing a variety of novel soft systems that were inconceivable just a decade or two ago. ${ }^{15,27,109}$ This is, however, not all fun and games. There are still certain aspects of the particle preparation that require better understanding. The synthesis of hematite colloids itself is very peculiar. While it seems straightforward in its execution, two identical bottles containing the same reaction mixture placed in the oven at the same time can give significantly different results, a problem that we are just beginning to shed light on. Furthermore, having a clearer picture of how the hematite microparticles grow can open the route to novel shapes which in turn can lead to novel applications. So far, works reporting magnetic hematite microparticles as building units mostly focus on the simplest, and most commonly known shapes such as ellipsoidal and peanut-like, with an even stronger focus on the cubic shape; however new possibilities arising from the use of other shapes are tremendous especially when these magnetic particles are used in combination with other materials, ${ }^{15,107,109}$ packed in confinement, ${ }^{77,142,143}$ activated by chemical ${ }^{27,138,139}$ and magnetic ${ }^{9,17,18,144}$ stimuli or any combination of these. ${ }^{31}$ Even so, this is just the beginning. We have seen how the photocatalytic properties of hematite microparticles have lead to the development of unique active and responsive systems that can be produced cheaply and in bulk. The use of hematite particles has enabled, for instance, the design of chiral ${ }^{18}$ and active ${ }^{17}$ fluids, and the modeling of amorphous materials into well-defined shapes. ${ }^{21,22,106}$ Understanding how these processes work and can be controlled could smooth the path to active and responsive systems, bringing advantages in fields beyond soft matter. There is still a lot of work that can be done to understand how we can better control the properties of hematite microparticles; a challenge that can provide opportunities towards the design of novel active, adaptable and responsive soft materials.

\section{Conflicts of interest}

There are no conflicts to declare.

\section{Acknowledgements}

We thank Joe Donaldson for critically reading the manuscript. J. M. M. acknowledges financial support from the Netherlands Organisation for Scientific Research (NWO) (016.Veni.192.119).

\section{References}

1 E. Matijević and P. Scheiner, J. Colloid Interface Sci., 1978, 63, 509-524. 
2 T. Sugimoto and K. Sakata, J. Colloid Interface Sci., 1992, 152, 587-590.

3 M. Ozaki, H. Suzuki, K. Takahashi and E. Matijević, J. Colloid Interface Sci., 1986, 113, 76-80.

4 M. Ozaki, T. Egami, N. Sugiyama and E. Matijević, J. Colloid Interface Sci., 1988, 126, 212-219.

5 G. Muench, S. Arajs and E. Matijević, IEEE Trans. Magn., 1982, 18, 1583-1585.

6 G. J. Muench, S. Arajs and E. Matijević, J. Appl. Phys., 1998, 52, 2493-2495.

7 A. H. Morrish, Canted antiferromagnetism: hematite, World Scientific, Singapore, 1994, vol. 234.

8 W. Lowrie, Fundamentals of Geophysics, Cambridge University Press, Cambridge, UK, 2nd edn, 2007.

9 F. Martinez-Pedrero, A. Cebers and P. Tierno, Soft Matter, 2016, 12, 3688-3695.

10 S. H. Lee and C. M. Liddell, Small, 2009, 5, 1957-1962.

11 J.-M. Meijer, D. V. Byelov, L. Rossi, A. Snigirev, I. Snigireva, A. P. Philipse and A. V. Petukhov, Soft Matter, 2013, 9, 10729-10738.

12 L. Rossi, J. G. Donaldson, J.-M. Meijer, A. V. Petukhov, D. Kleckner, S. S. Kantorovich, W. T. M. Irvine, A. P. Philipse and S. Sacanna, Soft Matter, 2018, 14, 1080-1087.

13 A. Pal, T. Zinn, M. A. Kamal, T. Narayanan and P. Schurtenberger, Small, 2018, 14, 1802233.

14 M. Aoshima, M. Ozaki and A. Satoh, J. Phys. Chem. C, 2012, 116, 17862-17871.

15 S. Sacanna, L. Rossi and D. Pine, J. Am. Chem. Soc., 2012, 134, 6112-6115.

16 M. Youssef, T. Hueckel, G.-R. Yi and S. Sacanna, Nat. Commun., 2016, 7, 12216-12217.

17 M. Driscoll, B. Delmotte, M. Youssef, S. Sacanna, A. Donev and P. Chaikin, Nat. Phys., 2016, 13, 375-379.

18 V. Soni, E. S. Bililign, S. Magkiriadou, S. Sacanna, D. Bartolo, M. J. Shelley and W. T. M. Irvine, Nat. Phys., 2019, 15, 1188-1194.

19 H. Xie, M. Sun, X. Fan, Z. Lin, W. Chen, L. Wang, L. Dong and Q. He, Sci. Robot., 2019, 4, eaav8006.

20 S. H. Lee, Y. Song, I. D. Hosein and C. M. Liddell, J. Mater. Chem., 2009, 19, 350.

21 S. Lee, S. Gerbode, B. John, A. Wolfgang, F. Escobedo, I. Cohen and C. Liddell, J. Mater. Chem., 2008, 18, 4912-4916.

22 L. Rossi, S. Sacanna, W. T. M. Irvine, P. M. Chaikin, D. J. Pine and A. P. Philipse, Soft Matter, 2011, 7, 4139-4142.

23 L. Rossi, V. Soni, D. J. Ashton, D. J. Pine, A. P. Philipse, P. M. Chaikin, M. Dijkstra, S. Sacanna and W. T. M. Irvine, Proc. Natl. Acad. Sci. U. S. A., 2015, 112, 5286-5290.

24 J.-M. Meijer, V. Meester, F. Hagemans, H. N. W. Lekkerkerker, A. P. Philipse and A. V. Petukhov, Langmuir, 2019, 35, 4946-4955.

25 J.-M. Meijer, A. Pal, S. Ouhajji, H. N. W. Lekkerkerker, A. P. Philipse and A. V. Petukhov, Nat. Commun., 2017, 8, 14352.
26 J.-M. Meijer, F. Hagemans, L. Rossi, D. V. Byelov, S. I. R. Castillo, A. Snigirev, I. Snigireva, A. P. Philipse and A. V. Petukhov, Langmuir, 2012, 28, 7631-7638.

27 J. Palacci, S. Sacanna, A. P. Steinberg, D. J. Pine and P. M. Chaikin, Science, 2013, 339, 936-940.

28 J. Palacci, S. Sacanna, A. Vatchinsky, P. M. Chaikin and D. J. Pine, J. Am. Chem. Soc., 2013, 135, 15978-15981.

29 J. Palacci, S. Sacanna, S. H. Kim, G.-R. Yi, D. J. Pine and P. M. Chaikin, Philos. Trans. R. Soc., A, 2014, 372, 20130372.

30 A. Aubret, M. Youssef, S. Sacanna and J. Palacci, Nat. Phys., 2018, 14, 1114-1118.

31 A. Aubret and J. Palacci, Soft Matter, 2018, 14, 9577-9588.

32 J. K. Bailey, C. Brinker and M. L. Mecartney, J. Colloid Interface Sci., 1993, 157, 1-13.

33 M. Ozaki, S. Kratohvil, N. Sugiyama and E. Matijević, J. Colloid Interface Sci., 1984, 102, 146-151.

34 M. Ocana, M. Morales and C. Serna, J. Colloid Interface Sci., 1999, 212, 317-323.

35 T. P. Raming, A. Winnubst, C. M. van Kats and A. P. Philipse, J. Colloid Interface Sci., 2002, 249, 346-350.

36 C. Wu, P. Yin, X. Zhu, C. OuYang and Y. Xie, The, J. Phys. Chem. B, 2006, 110, 17806-17812.

37 Y. Huo, Y. Zhu, J. Xie, G. Cao, T. Zhu, X. Zhao and S. Zhang, RSC Adv., 2013, 3, 19097-19103.

38 M. Tadic, M. Panjan, V. Damnjanovic and I. Milosevic, Appl. Surf. Sci., 2014, 320, 183-187.

39 T. Wang, S. Zhou, C. Zhang, J. Lian, Y. Liang and W. Yuan, New J. Chem., 2014, 38, 46-49.

40 D. Cardillo, M. Tehei, M. S. Hossain, M. M. Islam, K. Bogusz, D. Shi, D. Mitchell, M. Lerch, A. Rosenfeld, S. Corde and K. Konstantinov, ACS Appl. Mater. Interfaces, 2016, 8, 5867-5876.

41 T. P. Almeida, M. Fay, Y. Zhu and P. D. Brown, The, J. Phys. Chem. C, 2009, 113, 18689-18698.

42 M. Reufer, H. Dietsch, U. Gasser, B. Grobety, A. M. Hirt, V. K. Malik and P. Schurtenberger, J. Phys.: Condens. Matter, 2011, 23, 065102.

43 V. Malik, B. Grobety, V. Trappe, H. Dietsch and P. Schurtenberger, Colloids Surf., A, 2014, 445, 21-29.

44 A. Navrotsky, L. Mazeina and J. Majzlan, Science, 2008, 319, 1635-1638.

45 S. Hamada and E. Matijević, J. Colloid Interface Sci., 1981, 84, 274-277.

46 S. Hamada and E. Matijević, J. Chem. Soc., Faraday Trans. 1, 1982, 78, 2147-2156.

47 S. Hamada, S. Niizeki and Y. Kudo, Bull. Chem. Soc. Jpn., 1986, 59, 3443-3450.

48 K. Kandori, A. Yasukawa and T. Ishikawa, J. Colloid Interface Sci., 1996, 180, 446-452.

49 K. Kandori, N. Yamamoto, A. Yasukawa and T. Ishikawa, Phys. Chem. Chem. Phys., 2002, 4, 6116-6122.

50 K. Kandori, Y. Yamoto and T. Ishikawa, J. Colloid Interface Sci., 2005, 283, 432-439.

51 K. Kandori and M. Watanabe, Colloid Polym. Sci., 2011, 289, 981-991.

52 F. Zhang, H. Yang, X. Xie, L. Li, Z. Lihui, J. Yu, H. Zhao and B. Liu, Sens. Actuators, B, 2009, 141, 381-389. 
53 J. Lian, X. Duan, J. Ma, P. Peng, T. Kim and W. Zheng, ACS Nano, 2009, 3, 3749-3761.

54 J. Ma, J. Lian, X. Duan, X. Liu and W. Zheng, The, J. Phys. Chem. C, 2010, 114, 10671-10676.

55 N. K. Chaudhari, H. C. Kim, C. S. Kim, J. Park and J.-S. Yu, CrystEngComm, 2012, 14, 2024-2031.

56 G. Xu, L. Li, Z. Shen, Z. Toa, Y. Zhang, H. Tian, X. Wei, G. Shen and G. Han, J. Alloys Compd., 2015, 629, 36-42.

57 Z. Li, G. Meng, R. Chen and X. Song, RSC Adv., 2015, 5, 88787-88795.

58 T. Sugimoto, M. M. Khan and A. Muramatsu, Colloids Surf., A, 1993, 70, 167-169.

59 T. Sugimoto, S. Waki, H. Itoh and A. Muramatsu, Colloids Surf., A, 1996, 109, 155-165.

60 T. Sugimoto, H. Itoh and T. Mochida, J. Colloid Interface Sci., 1998, 205, 42-52.

61 R. Al-Gaashani, S. Radiman, N. Tabet and A. R. Daud, J. Alloys Compd., 2013, 550, 395-401.

62 E. Matijević, Acc. Chem. Res., 1981, 14, 22-29.

63 M. Ozaki, N. Ookoshi and E. Matijević, J. Colloid Interface Sci., 1990, 137, 546-549.

64 K. Kandori, S. Tamura and T. Ishikawa, Colloid Polym. Sci., 1994, 272, 812-819.

65 T. Sugimoto and Y. Wang, J. Colloid Interface Sci., 1998, 207, 137-149.

66 B. Jia and L. Gao, Cryst. Growth Des., 2008, 8, 1372-1376.

67 T. Sugimoto, K. Sakata and A. Muramatsu, J. Colloid Interface Sci., 1993, 159, 372-382.

68 T. Sugimoto, M. M. Khan, A. Muramatsu and H. Itoh, Colloids Surf., A, 1993, 79, 233-247.

69 B. Hou, Y. Wu, L. Wu, Y. Shi, K. Zou and H. Gai, Mater. Lett., 2006, 60, 3188-3191.

70 R. Zha, T. Shi, Z. Zhang, D. Xu, T. Jiang and M. Zhang, RSC Adv., 2017, 7, 6345-6357.

71 T. Sugimoto and A. Muramatsu, J. Colloid Interface Sci., 1996, 184, 626-638.

72 N. Sasaki, Y. Murakami, D. Shindo and T. Sugimoto, J. Colloid Interface Sci., 1999, 213, 121-125.

73 M. Ocaña, R. Rodriguez-Clemente and C. J. Serna, Adv. Mater., 1995, 7, 212-216.

74 C. Frandsen, B. A. Legg, L. R. Comolli, H. Zhang, B. Gilbert, E. Johnson and J. F. Banfield, CrystEngComm, 2014, 16, 1451-1458.

75 T. Sugimoto, Y. Wang, H. Itoh and A. Muramatsu, Colloids Surf., A, 1998, 134, 265-279.

76 E. Matijević, Annu. Rev. Mater. Sci., 1985, 15, 483-516.

77 J. W. J. de Folter, P. Liu, L. Jiang, A. Kuijk, H. E. Bakker, A. Imhof, A. van Blaaderen, J. Huang, W. K. Kegel, A. P. Philipse and A. V. Petukhov, Part. Part. Syst. Charact., 2014, 32, 313-320.

78 T. G. Anjali and M. G. Basavaraj, Phys. Chem. Chem. Phys., 2017, 19, 30790-30797.

79 G. Park, D. Shindo, Y. Waseda and T. Sugimoto, J. Colloid Interface Sci., 1996, 177, 198-207.

80 D. Shindo, G. S. Park, Y. Waseda and T. Sugimoto, J. Colloid Interface Sci., 1994, 168, 478-484.
81 D. Shindo, B.-T. Lee, Y. Waseda, A. Muramatsu and T. Sugimoto, Mater. Trans., JIM, 1993, 34, 580-585.

82 N. R. Jana, Y. Chen and X. Peng, Chem. Mater., 2004, 16, 3931-3935.

83 I. Dzyaloshinsky, J. Phys. Chem. Solids, 1958, 4, 241-255.

84 T. Moriya, Phys. Rev., 1960, 120, 91-98.

85 F. J. Morin, Phys. Rev., 1950, 78, 819-820.

86 A. H. Morrish, G. B. Johnston and N. A. Curry, Phys. Lett., 1963, 7, 177-178.

87 R. Nathans, S. J. Pickart, H. A. Alperin and P. J. Brown, Phys. Rev., 1964, 136, A1641-A1647.

88 E. Krén, B. Molnàr, E. Svàb and É. Zsoldos, Solid State Commun., 1974, 15, 1707-1710.

89 D. Kim, N. Lee, M. Park, B. H. Kim, K. An and T. Hyeon, J. Am. Chem. Soc., 2009, 131, 454-455.

90 I. Martchenko, J. J. Crassous, A. M. Mihut, E. Bialik, A. M. Hirt, C. Rufier, A. Menzel, H. Dietsch, P. Linse and P. Schurtenberger, Soft Matter, 2016, 12, 8755-8767.

91 C. Dagallier, H. Dietsch, P. Schurtenberger and F. Scheffold, Soft Matter, 2010, 6, 2174-2175.

92 Heterogeneous Photocatalysis, ed. J. C. Colmenares and Y.-J. $\mathrm{Xu}$, Springer-Verlag, Berlin Heidelberg, 2016.

93 Chemical Physics of Nanostructured Semiconductors, ed. A. I. Kokorin, CRC Press, Utrecht, Boston, 2003.

94 X. Kang, S. Liu, Z. Dai, Y. He, X. Song and Z. Tan, Catalysts, 2019, 9(2), 191.

95 J. K. Leland and A. J. Bard, J. Phys. Chem., 2015, 91, 1-8.

$96 \mathrm{~J}$. Li and D. Chu, in Multifunctional Photocatalytic Materials for Energy, ed. Z. Lin, M. Ye and M. Wang, Woodhead Publishing, 2018, pp. 49-78.

97 C. M. Eggleston, Science, 2008, 320, 184-185.

98 D. K. Bora, A. Braun and E. C. Constable, Energy Environ. Sci., 2013, 6, 407-425.

99 A. G. Tamirat, J. Rick, A. A. Dubale, W.-N. Su and B.-J. Hwang, Nanoscale Horiz., 2016, 1, 243-267.

100 K. R. Tolod, S. Hernández, E. A. Quadrelli and N. Russo, Visible Light-Driven Catalysts for Water Oxidation: Towards Solar Fuel Biorefineries, Elsevier B.V., 1st edn, 2019, vol. 178.

101 T. Wang, Z. Luo, C. Li and J. Gong, Chem. Soc. Rev., 2014, 43, 7469-7484.

102 J. A. Klug, N. G. Becker, S. C. Riha, A. B. F. Martinson, J. W. Elam, M. J. Pellin and T. Proslier, J. Mater. Chem. A, 2013, 1, 11607.

103 Y. Ling, G. Wang, D. A. Wheeler, J. Z. Zhang and Y. Li, Nano Lett., 2011, 11, 2119-2125.

104 M. Barroso, A. J. Cowan, S. R. Pendlebury, M. Grätzel, D. R. Klug and J. R. Durrant, J. Am. Chem. Soc., 2011, 133, 14868-14871.

105 S. J. Gerbode, S. H. Lee, C. M. Liddell and I. Cohen, Phys. Rev. Lett., 2008, 101, 058302.

106 Y. Wang, X. Su, P. Ding, S. Lu and H. Yu, Langmuir, 2013, 29, 11575-11581.

107 T. Hueckel and S. Sacanna, ACS Nano, 2018, 12, 3533-3540.

108 S. I. R. Castillo, N. A. Krans, C. E. L. Pompe, J. H. A. den Otter, D. M. E. Thies-Weesie and A. P. Philipse, Colloids Surf., A, 2016, 504, 228-233. 
109 M. Youssef, T. Hueckel, G.-R. Yi and S. Sacanna, Nat. Commun., 2019, 1-7.

110 J. H. Kim, H. J. Hwang, J. S. Oh, S. Sacanna and G.-R. Yi, J. Am. Chem. Soc., 2018, 140, 9230-9235.

111 Z. Bin, D. Li, Y. Long and K. Song, Sci. Rep., 2019, 1-8.

112 M. Ohmori and E. Matijevic, J. Colloid Interface Sci., 1992, 150, 594-598.

113 S. Sacanna, L. Rossi, B. W. M. Kuipers and A. P. Philipse, Langmuir, 2006, 22, 1822-1827.

114 S. Sacanna, L. Rossi and A. P. Philipse, Langmuir, 2007, 23, 9974-9982.

115 X. Xu and M. B. Cortie, J. Phys. Chem. C, 2007, 111, 18135-18142.

116 X. W. Lou, C. Yuan and L. A. Archer, Adv. Mater., 2007, 19, 3328-3332.

117 J. J. Crassous, A. M. Mihut, H. Dietsch, O. Pravaz, L. Ackermann-Hirschi, A. M. Hirt and P. Schurtenberger, Nanoscale, 2014, 6, 8726-8735.

118 Y. Wang, Y. Wang, D. R. Breed, V. N. Manoharan, L. Feng, A. D. Hollingsworth, M. Weck and D. J. Pine, Nature, 2012, 491, 51-55.

119 Q. Chen, S. C. Bae and S. Granick, Nature, 2011, 469, 381-384.

120 J. A. Diaz A, J. S. Oh, G.-R. Yi and D. J. Pine, Proc. Natl. Acad. Sci. U. S. A., 2020, 117, 10645-10653.

121 D. J. Kraft, R. Ni, F. Smallenburg, M. Hermes, K. Yoon, D. A. Weitz, A. van Blaaderen, J. Groenewold, M. Dijkstra and W. K. Kegel, Proc. Natl. Acad. Sci. U. S. A., 2012, 109, 10787-10792.

122 S. Sacanna, W. T. M. Irvine, P. M. Chaikin and D. J. Pine, Nature, 2010, 464, 575-578.

123 K. Han, C. W. Shields, N. M. Diwakar, B. Bharti, G. P. López and O. D. Velev, Sci. Adv., 2017, 3, e1701108.

124 H. Massana-Cid, F. Meng, D. Matsunaga, R. Golestanian and P. Tierno, Nat. Commun., 2019, 10, 1-8.

125 P. Tierno, Phys. Chem. Chem. Phys., 2014, 16, 23515-23528.

126 M. A. Kamal, A. V. Petukhov and A. Pal, J. Phys. Chem. B, 2020, 124, 5754-5760.
127 F. Martinez-Pedrero, A. Cebers and P. Tierno, Phys. Rev. Appl., 2016, 6, 034002.

128 S. Kantorovich, R. Weeber, J. J. Cerdà and C. Holm, J. Magn. Magn. Mater., 2011, 323, 1269-1272.

129 S. Kantorovich, R. Weeber, J. J. Cerdà and C. Holm, Soft Matter, 2011, 7, 5217-5227.

130 A. M. Mihut, A. Sánchez-Ferrer, J. J. Crassous, L. A. Hirschi, R. Mezzenga and H. Dietsch, Polymer, 2013, 54, 4194-4203.

131 M. Reufer, H. Dietsch, U. Gasser, A. Hirt, A. Menzel and P. Schurtenberger, J. Phys. Chem. B, 2010, 114, 4763-4769.

132 V. Städele, U. Gasser and H. Dietsch, Soft Matter, 2012, 8, 4427-4431.

133 C. Dagallier, F. Cardinaux, H. Dietsch and F. Scheffold, Soft Matter, 2012, 8, 4067-4071.

134 D. Hoffelner, M. Kundt, A. M. Schmidt, E. Kentzinger, P. Bender and S. Disch, Faraday Discuss., 2015, 181, 449-461.

135 I. Martchenko, J. J. Crassous, A. M. Mihut, E. Bialik, A. M. Hirt, C. Rufier, A. Menzel, H. Dietsch, P. Linse and P. Schurtenberger, Soft Matter, 2016, 12, 8755-8767.

136 D. Zákutná, Y. Falke, D. Dresen, S. Prévost, P. Bender, D. Honecker and S. Disch, Nanoscale, 2019, 11, 7149-7156.

137 A. Pal, V. A. Martinez, T. H. Ito, J. Arlt, J. J. Crassous, W. C. K. Poon and P. Schurtenberger, Sci. Adv., 2020, 6, eaaw9733.

138 S. Ramananarivo, É. Ducrot and J. Palacci, Nat. Commun., 2019, 10, 1-8.

139 H. Massana-Cid, J. Codina, I. Pagonabarraga and P. Tierno, Proc. Natl. Acad. Sci. U. S. A., 2018, 115, 10618-10623.

140 H. Moyses, J. Palacci, S. Sacanna and D. G. Grier, Soft Matter, 2016, 12, 6357-6364.

141 Z. Lin, X. Fan, M. Sun, C. Gao, Q. He and H. Xie, ACS Nano, 2018, 12, 2539-2545.

142 E. G. Teich, G. van Anders, D. Klotsa, J. Dshemuchadse and S. C. Glotzer, Proc. Natl. Acad. Sci. U. S. A., 2016, 113, E669-E678.

143 V. Manoharan and D. Pine, MRS Bull., 2004, 29, 91-95.

144 F. Martinez-Pedrero and P. Tierno, Phys. Rev. Appl., 2015, 3, 051003. 